\title{
Design and Simulation of Next-Generation High-Power, High-Brightness Laser Diodes
} \author{
Gaëlle; Georges, Patrick; MacKenzie, Roderick C.I.; Bream, Philip \\ Total number of authors: \\ 20 \\ Published in: \\ IEEE Journal of Selected Topics in Quantum Electronics \\ Link to article, DOI: \\ 10.1109/JSTQE.2008.2011286 \\ Publication date: \\ 2009 \\ Document Version \\ Publisher's PDF, also known as Version of record
}

Lim, Jun Jun; Sujecki, Slawomir; Lang, Lei; Zhang, Zichao; Paboeuf, David; Pauliat, Gilles; Lucas-Leclin,

Link back to DTU Orbit

Citation (APA):

Lim, J. J., Sujecki, S., Lang, L., Zhang, Z., Paboeuf, D., Pauliat, G., Lucas-Leclin, G., Georges, P., MacKenzie, R. C. I., Bream, P., Bull, S., Hasler, K-H., Sumpf, B., Wenzel, H., Erbert, G., Thestrup Nielsen, B., Petersen, P. M., Michel, N., Krakowski, M., \& Larkins, E. C. (2009). Design and Simulation of Next-Generation High-Power, High-Brightness Laser Diodes. IEEE Journal of Selected Topics in Quantum Electronics, 15(3), 993-1008. https://doi.org/10.1109/JSTQE.2008.2011286

\section{General rights}

Copyright and moral rights for the publications made accessible in the public portal are retained by the authors and/or other copyright owners and it is a condition of accessing publications that users recognise and abide by the legal requirements associated with these rights.

- Users may download and print one copy of any publication from the public portal for the purpose of private study or research.

- You may not further distribute the material or use it for any profit-making activity or commercial gain

- You may freely distribute the URL identifying the publication in the public portal 


\title{
Design and Simulation of Next-Generation High-Power, High-Brightness Laser Diodes
}

\author{
Jun Jun Lim, Member, IEEE, Slawomir Sujecki, Senior Member, IEEE, Lei Lang, Student Member, IEEE, \\ Zhichao Zhang, David Paboeuf, Gilles Pauliat, Gaëlle Lucas-Leclin, Patrick Georges, Roderick C. I. MacKenzie, \\ Philip Bream, Stephen Bull, Member, IEEE, Karl-Heinz Hasler, Bernd Sumpf, Hans Wenzel, \\ Götz Erbert, Member, IEEE, Birgitte Thestrup, Paul Michael Petersen, Nicolas Michel, Michel Krakowski, \\ and Eric C. Larkins, Senior Member, IEEE
}

(Invited Paper)

\begin{abstract}
High-brightness laser diode technology is progressing rapidly in response to competitive and evolving markets. The large volume resonators required for high-power, high-brightness operation makes their beam parameters and brightness sensitive to thermal- and carrier-induced lensing and also to multimode operation. Power and beam quality are no longer the only concerns for the design of high-brightness lasers. The increased demand for these technologies is accompanied by new performance requirements, including a wider range of wavelengths, direct electrical modulation, spectral purity and stability, and phase-locking techniques for coherent beam combining. This paper explores some of the next-generation technologies being pursued, while illustrating the growing importance of simulation and design tools. The paper begins by investigating the brightness limitations of broadarea laser diodes, including the use of asymmetric feedback to improve the modal discrimination. Next, tapered lasers are considered, with an emphasis on emerging device technologies for applications requiring electrical modulation and high spectral brightness.
\end{abstract}

Manuscript received November 8, 2008; revised December 2, 2008. First published March 6, 2009; current version published June 5, 2009. This work was supported in part by the European Commission under Project WWW.BRIGHTER.EU (IST-035266), Project WWW.BRIGHT.EU (IST-511722), Project ULTRABRIGHT (IST-1999-10356), and Project POWERPACK (IST-2000-29447). The work of R. MacKenzie and P. J. Bream was supported by the Engineering and Physical Sciences Research Council, U.K. The work of D. Paboeuf was supported by the French Ministry of Defense (Délégation Générale de l'Armement).

J. J. Lim, S. Sujecki, L. Lang, Z. Zhang, R. MacKenzie, P. Bream, S. Bull and E. C. Larkins are with the Department of Electrical and Electronic Engineering, University of Nottingham, Nottingham NG7 2RD, U.K. (e-mail: jun.lim@nottingham.ac.uk; slawomir.sujecki@nottingham. ac.uk; eric.larkins@ @ottingham.ac.uk).

D. Paboeuf, G. Pauliat, G. Lucas-Leclin, and P. Georges are with the Laboratoire Charles Fabry de l'Institut d'Optique, Centre National de la Recherche Scientifique (CNRS), University Paris Sud, 91127 Palaiseau, France (e-mail: david.paboeuf@institutoptique.fr; gilles.pauliat@iota.u-psud.fr; gaelle.lucasleclin@institutoptique.fr; patrick.georges@iota.u-psud.fr).

K.-H. Hasler, B. Sumpf, H. Wenzel, and G. Erbert are with the FerdinandBraun-Institut für Höchstfrequenztechnik, 12489 Berlin, Germany (e-mail: karl-heinz.hasler@fbh-berlin.de; bernd.sumpf@fbh-berlin.de; hans.wenzel@ fbh-berlin.de; goetz.erbert@fbh-berlin.de).

B. Thestrup and P. M. Petersen are with the DTU Fotonik, Technical University of Denmark, 4000 Roskilde, Denmark (e-mail: birgitte.thestrup@ risoe.dk; paul.michael.petersen@ risoe.dk).

N. Michel and M. Krakowski are with Alcatel-Thales III-V Lab, 91767 Palaiseau, France (e-mail: nicolas.michel@3-5lab.fr; michel.krakowski@35lab.fr).

Color versions of one or more of the figures in this paper are available online at http://ieeexplore.ieee.org.

Digital Object Identifier 10.1109/JSTQE.2008.2011286
These include two-contact lasers, self-organizing cavity lasers, and a phase-locked laser array using an external Talbot cavity.

Index Terms-External cavity laser diodes, high-brightness lasers, high-power lasers, laser simulation, modal discrimination, phasecoupled laser diode arrays, self-organizing laser.

\section{INTRODUCTION}

$\mathbf{T}$ HERE is a growing demand for high-power diode lasers in a variety of fields today. Traditionally, the largest application for high-power diode lasers has been as pump sources for solid-state lasers, fiber amplifiers in telecommunications, and more recently, fiber lasers [1]. Diode lasers are used because they have a high electrical to optical conversion efficiency and can be designed to fit the absorption band of these applications. They have advantages in terms of reliability, compactness, and cost. In recent years, high-brightness laser technology has been strongly driven by an increasing number and variety of applications, including medicine (e.g., photodynamic therapy, fluorescence spectroscopy, and surgery), display technology (laser displays and mobile projectors), free-space optical wireless communication, and direct-diode materials processing.

As the range of applications increases, the performance demands on high-brightness laser diodes are also becoming more stringent. Performance requirements for next-generation highbrightness laser diodes include good modulation performance, narrow spectral linewidth, and nearly diffraction-limited powers of 20-100 W. At the same time, laser simulation tools are becoming essential for achieving the desired performance. These tools provide an understanding of the physics and operation of the device and permit the exploration of novel designs and concepts needed to provide more than an incremental improvement in the device performance.

In this paper, we provide an overview of recent trends in the design and simulation of next-generation high-power, highbrightness laser diodes. Section II begins with a cursory discussion of the applications, performance requirements, and current state of the art of high-brightness diode lasers and systems, followed by an overview of approaches for achieving high power and brightness, and a discussion of the increased role and demands on simulation and design tools. Section III briefly describes the high-brightness laser diode simulation tools used in 


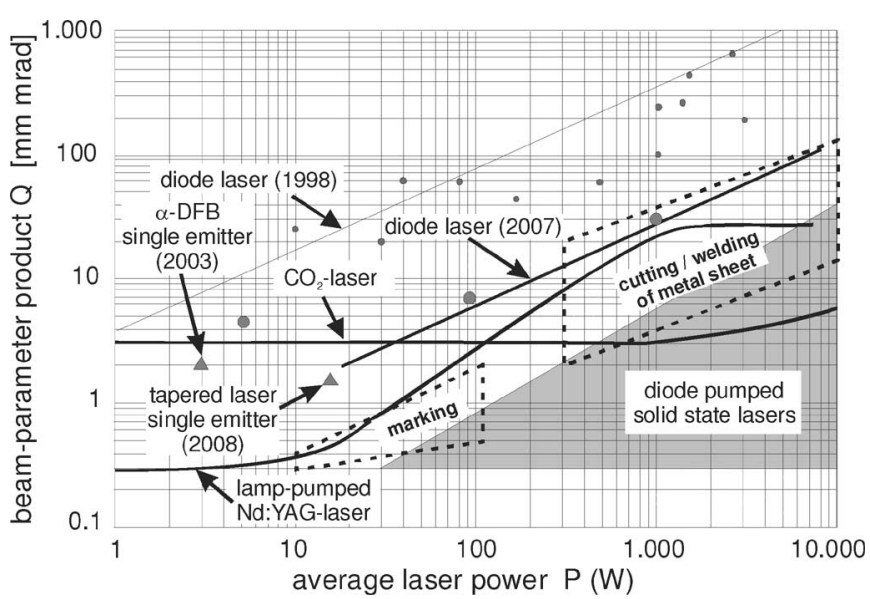

Fig. 1. Typical beam parameters for different laser systems and for applications in materials processing. (Courtesy P. Loosen, Fraunhofer Institute for Laser Technology.)

this paper and how the simulation parameters are calibrated and validated. Section IV considers a few examples to provide insight into the approaches and design considerations currently being pursued. These include an asymmetric feedback external cavity laser, a multiple section tapered laser, a self-organizing cavity laser, and a laser array in an external Talbot cavity.

\section{BACKGROUND}

\section{A. Applications and Performance Requirements}

The most important performance target for high-power laser diodes continues to be the combination of power and beam quality, known as brightness. The brightness of a laser beam is defined as the optical power density $P$ per emission area $A$ and per unit of solid angle $\Omega$ in the output beam. The brightness $B$ describes how well the optical power can be collimated into a narrow beam, and can be described as

$$
B=\frac{P}{A \Omega} \propto \frac{P}{Q^{2}}=\frac{P}{\left(\omega_{0} \theta_{f}\right)^{2}} \propto \frac{P}{\left(M^{2}\right)^{2}} .
$$

The brightness is also related to $M^{2}$ and the beam parameter product $Q$ (product of the minimum beam diameter $\omega_{0}$ and its divergence $\theta_{f}$ ). The current state of the art for power and brightness of diode lasers and systems is shown in Fig. 1. The comparison with other high-power lasers (e.g. $\mathrm{CO}_{2}$ and solidstate lasers) in Fig. 1 shows that diode lasers are approaching the level of power and brightness achievable by other laser systems.

As the variety of applications for high-brightness laser diodes increases, so does the range of performance specifications, which generally depend upon the intended application. For example, most high-brightness diode lasers operate at 808 or $980 \mathrm{~nm}$, but other wavelengths are emerging for applications such as medicine, displays, printing, and marking/cutting/welding of plastics. Display and optical wireless applications also require that the laser has a controlled emission spectrum and beam quality during high-frequency modulation $(0.1-1 \mathrm{GHz})$ and a large modulation efficiency (power/current ratio). Applications requiring frequency doubling (e.g., blue and green lasers for displays, blue/near-UV lasers for fluorescence spectroscopy) are driving the pursuit of ultimate brightness combined with spectral purity/stability. The efficiency of frequency doubling depends on the square of the optical electric field and requires a nearly diffraction-limited beam to achieve a high optical field intensity and good beam coherence. As indicated in Fig. 1, applications involving the direct processing of materials are driving the development of laser systems with high output power and brightness. These include marking $(P=10$ $100 \mathrm{~W}, Q=0.3-2 \mathrm{~mm} \cdot \mathrm{mrad})$, cutting, and welding of sheet metal $(P=0.3-10 \mathrm{~kW}, Q=2-100 \mathrm{~mm} \cdot \mathrm{mrad})$. Currently, the best power and brightness results for single emitters are from tapered lasers $(P=12 \mathrm{~W}, Q=1.5 \mathrm{~mm} \cdot \mathrm{mrad}[2])$. Generally, power levels greater than $10-20 \mathrm{~W}$ must be achieved by combining the beams from multiple emitters. For the most demanding high-power applications, the beams must be combined coherently through stable phase-locking of an array of emitters to achieve power densities $>10^{5} \mathrm{~W} / \mathrm{mm}^{2}$.

\section{B. Approaches for High Power and Brightness}

The simplest laser diode to fabricate is the broad-area laser diode (BA-LD), which is still widely used to provide high power with high efficiency. However, they are known to have a large slow-axis far-field divergence and poor beam qualityprimarily due to beam filamentation. This process has been studied intensively both experimentally and theoretically, e.g., in [3] and references therein. Spatial filamentation leads to a complicated multilobed near-field pattern, which negatively affects the brightness. Furthermore, filamentation is sometimes accompanied by unwanted periodic or chaotic variations in the laser power. Hence, BA-LDs are fundamentally unsuitable for high-brightness applications without some form of filtering to provide a mechanism for modal discrimination.

To overcome the deficiencies of the BA-LD, numerous designs have been proposed to increase the brightness of highpower laser diodes. One technique adopted from traditional nondiode lasers is the unstable resonator concept, such as lasers with a curved mirror resonator [4], [5]. Lasers with multiple sections have also been explored to increase the brightness, including master oscillator power amplifier (MOPA) lasers [6], tapered lasers [7], and bow-tie lasers [8]. The $\alpha$-DFB laser showed early promise [9], but has only achieved an output power of $3 \mathrm{~W}$ with $M^{2}=3.2(Q=2 \mathrm{~mm} \cdot \mathrm{mrad})$ from a single emitter [10], [11]. The far-field divergence of a BA-LD can also be reduced using asymmetric feedback from an external cavity [12], [13].

The easiest way to scale the power from laser diodes is to employ an array of emitters. To achieve high brightness, however, phase locking is needed to coherently combine their output beams. Common approaches include positive guiding [14] (evanescent-wave coupling) and antiguiding (leaky-wave coupling) arrays [15], with the latter showing stronger in-phase coupling. Phase locking can also be achieved through diffractive coupling using the Talbot effect. This can be done monolithically [16] or using an external cavity [17]. Another phase 
locking method using diffractive coupling employs a multimode interference (MMI) coupling section [18].

\section{Role of Simulation and Design Tools}

Numerous laser models have been reported in the literature. These models vary in complexity and have typically been developed to target specific applications. Early models ignored current spreading in the cladding layers of the device and typically solved the 1-D unipolar diffusion equation in the lateral direction. These tools were used to explain spatial hole burning and carrier lensing effects and were also applied to explain the filamentary nature of broad-area lasers [19]. Later, full 2-D cross-sectional models were introduced, which were borrowed from modeling techniques developed for silicon device simulators [20]-[22]. These 2-D cross-sectional laser models solved the electrical, thermal, and optical problems self-consistently, making them more accurate-especially for ridge waveguide (RW) lasers. However, since they only considered a single cross section, they were unsuitable for longitudinally nonuniform structures and high-power operation, where longitudinal spatial hole burning and carrier/thermal lensing effects are significant. Early models based on beam propagation methods (BPMs) were developed to handle nonuniform structures, but typically only solved the 1-D electrical problem in the lateral direction [23]- [25]. Sophisticated models were also introduced that solved the spatiotemporal dynamics of the lasers, but these models also used a 1-D electrical model in the lateral direction [26], [27]. Quasi-3-D models were introduced in [28] and [29], with the optical model separated into 1-D in the longitudinal direction and 2-D in the transverse cross section. The combination of the 2-D cross-section electrothermal model with the BPM was then introduced [30], [31]. By including the longitudinal direction and accounting for current and heat spreading effects, these models have become predictive and useful design tools for high-brightness lasers. By including the spectral and/or the dynamic properties of the laser, design tools are reaching new levels of accuracy and reliability.

\section{LaSer Diode Model AND Its Calibration}

Ideally, to capture the complete physical behavior of the device, laser models should include all three spatial dimensions of the device, as well as their spectral and/or dynamic behavior. Short design cycles are often needed to respond to market demands, so compromises must be made between accuracy and efficiency. Models with reduced dimensionality and/or complexity are often useful early in the design cycle, where the emphasis is on designing first-generation devices. For design optimization, it is important to model the device more accurately.

For advanced simulations and design optimization, the characteristics to be modeled (apart from the light-current characteristic) include the near- and far-field patterns, the $M^{2}$ beam quality factor, and the astigmatism-all as a function of current. In addition to these external device characteristics, internal properties such as the temperature and carrier and photon density distributions are needed for a physical understanding of the operation of the device. At very high power densities, even

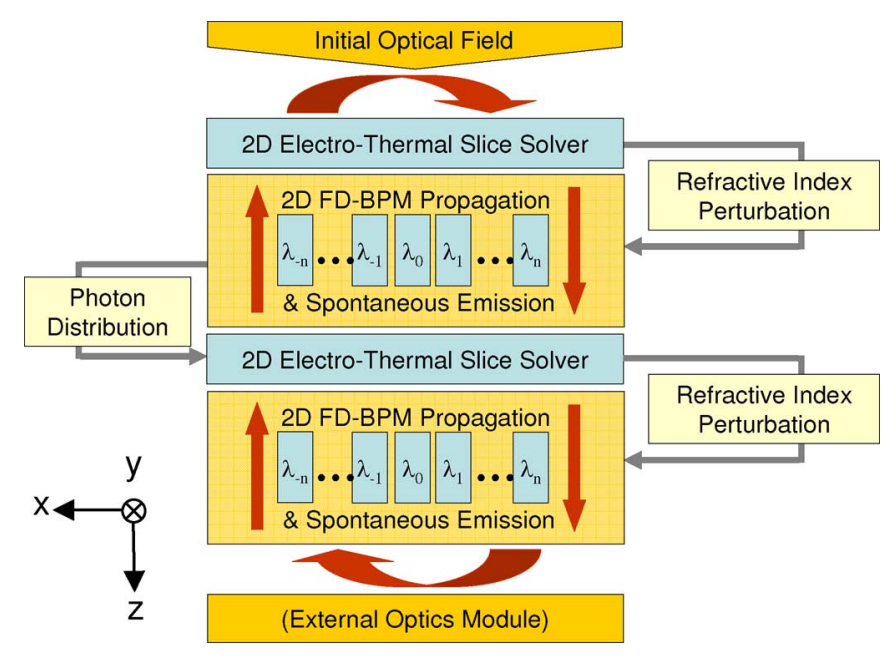

Fig. 2. Flow diagram of the 2.5-D spectral laser model.

nonequilibrium phenomena like carrier heating and spectral hole burning can be significant. This requires careful calibration of the materials parameters and extensive validation of the device models against measured results.

This section begins by describing our simulation tools for high-brightness laser diodes. This is followed by a description of initial calibration procedures and ends with a discussion of advanced validation for more comprehensive simulations.

\section{A. Description}

Our principal model for high-brightness lasers consists of a 2.5-D spectral laser model [32]. This model has been derived from a monochromatic 2.5-D laser model [33]. The term 2.5-D is used to indicate that the model is quasi-3-D, consisting of 2-D optical $(x-z)$ and electrothermal $(x-y)$ solvers (the flow diagram and definition of axes are given in Fig. 2). The flow of carriers and heat in the longitudinal direction is neglected. Comparison with a full 3-D electrothermal model [30], [31] has shown that this approximation is valid for structures that are slowly varying in the longitudinal direction (as investigated in this paper). The electrothermal model is coupled to the optical model through stimulated emission/absorption and spontaneous emission coupling and also through the carrier- and temperature-induced changes in the complex refractive index. The optical model propagates multiple wavelengths between electrothermal slices using the 2-D wide-angle finite-difference BPM (WA-FD-BPM) [34], the effective index approximation, and PML boundary conditions. The BPM projects the lateral modes onto the same field. Through mode competition, the multiwavelength model responds to both spectral and spatial variations in the gain distribution. The electrothermal and optical models are solved self-consistently, following an accelerated Fox-Li iterative approach [23].

The electrical model calculates the bipolar carrier density profiles for 2-D transverse slices along the laser cavity and includes drift-diffusion transport and the capture/escape processes between the bound and unbound states of the quantum well(s) (QWs). The thermal model, based upon the solution of the Boltzmann transport equation for heterostructures, solves 

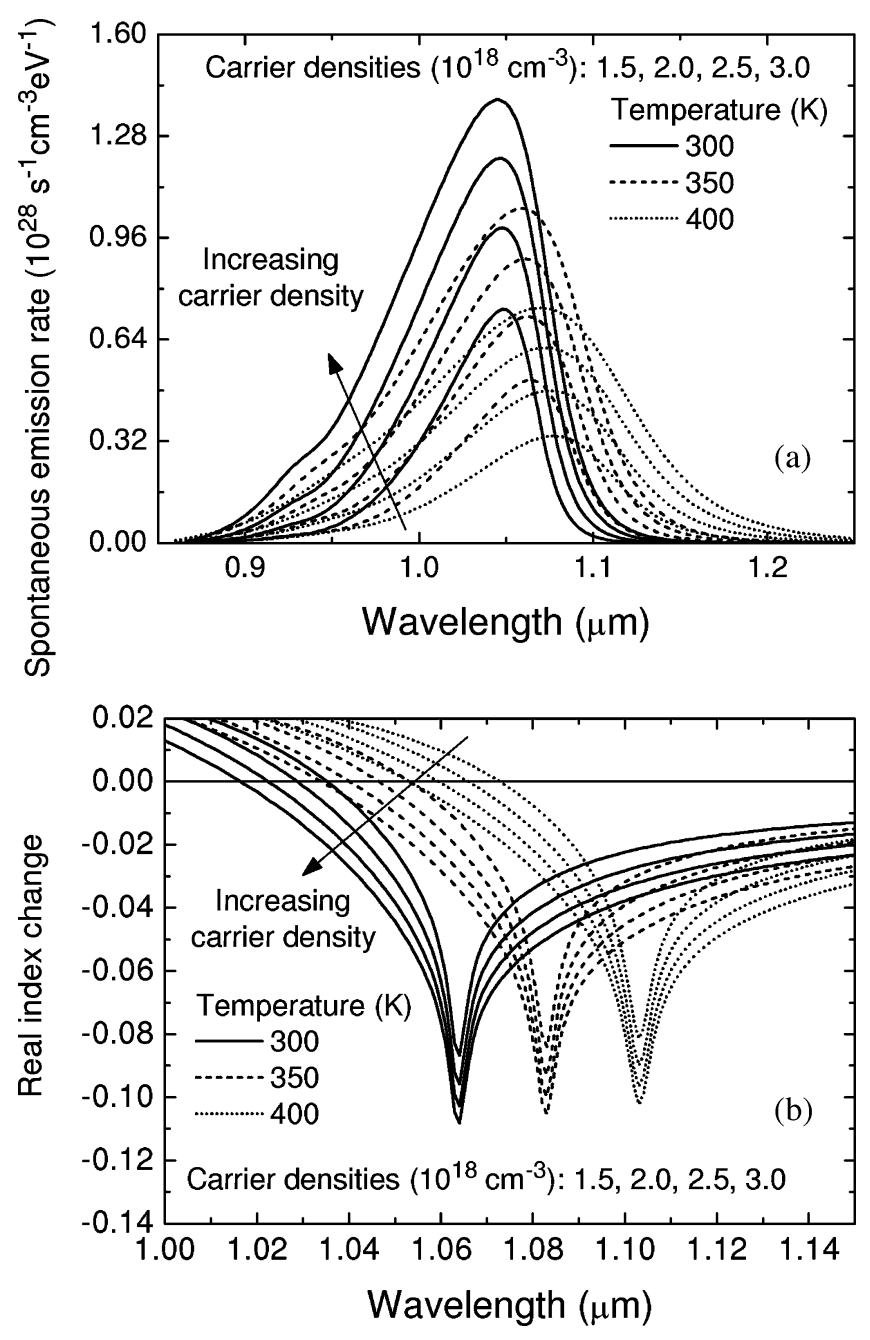

Fig. 3. (a) Spontaneous emission and (b) real index change spectra for a range of carrier densities and temperature.

the lattice heat flux equation (including thermal boundary resistances) and all relevant heat sources [35]. In addition, our thermal solver provides the option of including nonequilibrium effects by solving for the electron, hole, and longitudinal optical (LO) phonon temperatures in the QW active region(s) [36]. Surface recombination, Fermi level pinning, and surface depletion are included self-consistently [37], [38]. The photon density distribution at each wavelength is provided as an input to the electrical solver. The gain, spontaneous emission, and change in refractive index spectra, which depend on the electron and hole densities, temperature, and photon density, are parameterized in a look-up table and used by the optical model to propagate the fields to the next electrothermal slice.

An accurate spectral simulation requires a reliable model for the gain, spontaneous emission, and carrier-induced refractive index change. Our model uses the parabolic approximation for the conduction band and a $4 \times 4$ k.p band mixing model for the valence band to calculate the $\mathrm{QW}$ band structure. Examples of the spontaneous emission and carrier-induced index change spectra for a 1060-nm QW are shown in Fig. 3. The band param- eters were taken from the literature [39] and a band offset ratio of $\Delta E_{c} / \Delta E_{g}=0.65$ was used. The spontaneous emission spectra were calculated and convolved with a sech linewidth broadening function, as shown in Fig. 3(a). The intraband relaxation lifetime was taken to vary as a function of carrier density and temperature [40]. Next, the gain spectra were obtained by transforming the spontaneous emission spectra using Einstein's relation. The spectral change in the real index of the QW [Fig. 3(b)] was calculated with the Kramers-Kronig relation from the change in the calculated gain spectra, but does not include smaller changes due to intraband absorption. The effective index change is obtained by multiplying the QW index change by the confinement factor, but more accurate treatments should also account for index changes in the waveguide and cladding.

The 2.5-D spectral laser model can also be coupled to an external optics module to simulate external cavity lasers. This is particularly relevant for high-power lasers, where external cavities can be used to improve the beam quality of the laser. As the output facet reflectivity of a high-brightness laser diode is often $<1 \%$, this scheme can also be used to evaluate the impact of back reflections from the external optics.

Various simplifications of the 2.5-D spectral laser model exist to increase the efficiency of the simulation, depending on the application. These include isothermal, unipolar, monochromatic, and reduced dimensionality models, which are useful for obtaining qualitative results or when the size of the problem becomes computationally intractable. For example, the 1.5-D model is used in Section III to simulate large structures such as laser arrays and (in some cases) when the spectral characteristics of the device are required. The 1.5-D model is quasi-2-D in the sense that the optical problem is solved in the $x-z$ plane and the electrical problem is reduced to 1-D in the lateral $(x)$ direction. The 1-D electrical model solves the unipolar carrier-diffusion equation given as

$$
D_{a} \frac{d^{2}}{d x^{2}} n(x)=-\frac{J(x)}{q d}+R_{\mathrm{nr}}(x)+R_{\mathrm{spon}}(x)+v_{g} g(x) S(x)
$$

where $D_{a}, J, d, n, R_{\mathrm{nr}}, R_{\mathrm{spon}}, g$, and $S$ are the ambipolar diffusion coefficient, injection current density, active region thickness, carrier density, nonradiative recombination, spontaneous emission rate, optical gain, and photon density, respectively. The spontaneous emission rate and optical gain data are the same as those used in the 2-D bipolar electrical model for consistency and accuracy. In the 1-D electrical model, $J(x)$ is constant inside the stripe and zero outside the stripe.

The laser model used in this paper improves upon previous beam-propagation-based laser models [30], [31], [33] by solving the emission spectra of the laser and including the spectral dependence of the gain, spontaneous emission, and carrier-induced index change. It also has additional flexibility by including the option to couple to external optics modules to allow the simulation of a variety of external cavity lasers.

\section{B. Calibration Procedure}

Proper calibration is essential for a predictive laser model. A carefully calibrated simple model can be even more predictive 


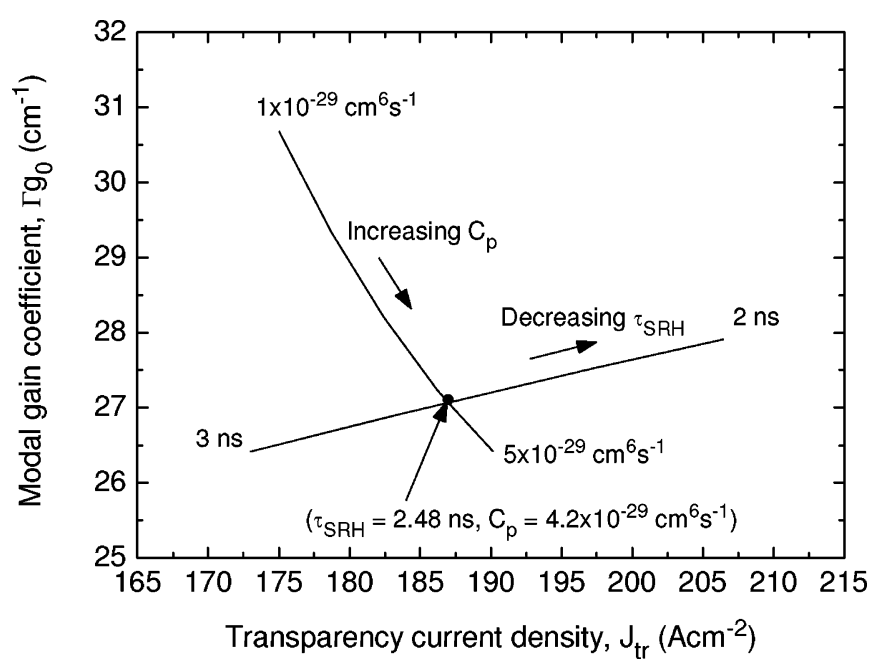

Fig. 4. $\Gamma g_{0}$ and $J_{\mathrm{tr}}$ dependence with $\tau_{\mathrm{SRH}}$ and $C_{p}$.

than a poorly calibrated sophisticated model. In this section, an example of the calibration of the laser model is presented for a device emitting at $1060 \mathrm{~nm}$ [41].

The first step is to calibrate the simulated gain and spontaneous emission spectra with gain spectra measured using the Hakki-Paoli [42], Cassidy [43], or segmented contact [44] methods. This usually involves slight adjustments to the QW energy levels and linewidth broadening. Next, the internal loss is determined. The internal loss can be obtained from lengthdependent measurements of the external differential efficiency of BA-LDs. For this structure, the internal loss $\alpha_{i}$ was $0.9 \mathrm{~cm}^{-1}$. At long wavelengths, the internal loss is dominated by intervalence band absorption. Therefore, the absorption cross section for holes in the QW was used as a fitting parameter and a value of $3 \times 10^{-17} \mathrm{~cm}^{2}$ gave an $\alpha_{i}$ that agreed with experiment.

The nonradiative recombination parameters are determined next-in particular, the Shockley-Read-Hall (SRH) carrier lifetime and the Auger recombination coefficient. These parameters can be obtained from length-dependent measurements of the threshold current of BA-LDs. From these measurements, the transparency current density $J_{\mathrm{tr}}$ and the modal gain coefficient $\Gamma g_{0}$ are extracted. Using the calculated gain and spontaneous recombination spectra, the SRH carrier lifetime and Auger recombination parameter were adjusted to obtain agreement with the experimental values of $\Gamma g_{0}$ and $J_{\mathrm{tr}}$. The SRH carrier lifetime for electrons and holes was set equal to each other $\left(\tau_{n}=\tau_{p}=\tau_{\mathrm{SRH}}\right)$. Only the Auger coefficient for holes $C_{p}$ was adjusted, as it is generally dominant at long wavelengths. By keeping $\tau_{\mathrm{SRH}}$ fixed and increasing $C_{p}$, we find that $\Gamma g_{0}$ decreases and $J_{\mathrm{tr}}$ increases according to the direction indicated by the line "increasing $C_{p}$ " in Fig. 4. Instead, if we keep $C_{p}$ fixed and decrease $\tau_{\mathrm{SRH}}, \Gamma g_{0}$ and $J_{\mathrm{tr}}$ both increase in the direction of the line labeled "decreasing $\tau_{\mathrm{SRH}}$ " in Fig. 4. If we search the entire parameter space of $\tau_{\mathrm{SRH}}$ and $C_{p}$, the set of recombination parameters $\tau_{\mathrm{SRH}}=2.48 \mathrm{~ns}$ and $C_{p}=4.2 \times 10^{-29} \mathrm{~cm}^{6} \mathrm{~s}^{-1}$ can be uniquely linked to the set of measured device parameters $\Gamma g_{0}=27.1 \mathrm{~cm}^{-1}$ and $J_{\mathrm{tr}}=187 \mathrm{~A} / \mathrm{cm}^{2}$.

\section{Advanced Validation for Accurate Device Simulation}

In this section, we discuss the general approach we follow for the experimental validation and calibration of more advanced simulation parameters. (Please note that the examples presented in Section IV represent very recent work, for which this validation is still being performed.)

The electrical and optical properties of laser diodes both depend upon temperature. Thus, their behavior is sensitive to selfheating, and it is important to validate the parameters used in the thermal model. As the rise in internal temperature usually causes a red-shift in emission wavelength above threshold [45]-[47], this can often be used to determine the thermal resistivity of the laser diode package. (Frequency-stabilized lasers are an exception.) Next, carrier- and temperature-induced lensing effects are investigated by comparing the simulated and measured near- and far-field profiles. Fig. 3(b) shows that the refractive index change near the ground state transition energy of the QW is highly sensitive to temperature. Thus, an a priori calculation of the refractive index change requires precise knowledge of the thermal environment (e.g., heat sink performance). Good agreement can usually be achieved by adjusting the proportionality constants relating the index change and changes in carrier density and temperature [30], [31], so that the model is able to predict the evolution of the far field with bias current with reasonable reliability.

Direct validation of the carrier distribution inside the cavity can be obtained from intracavity spontaneous emission measurements, which provide spectral and spatial information about the spontaneous emission distribution in the cavity. The intensity profiles reveal spatial hole burning and electrical overpumping effects. The spontaneous emission spectra provide information about the local lattice temperature, carrier heating, and spectral hole burning effects. Fig. 5(a) shows the spontaneous emission distribution obtained through a windowed contact in a 975-nm tapered laser [48]. Fig. 5(b) compares the simulated and experimental spontaneous emission distributions for a lateral slice near the output facet. The spatial hole in the center of the cavity observed in the experimental measurement is also seen in the simulation. Good quantitative agreement was also obtained. The difference in the integrated intensity was $\sim 12.4 \%$, which translates to $\sim 6 \%$ error in the carrier density.

Figs. 6 and 7 show experimental and simulated intracavity spontaneous emission spectra from the same device for different bias levels [32]. The measured spectra were taken from a $5 \mu \mathrm{m} \times 5 \mu \mathrm{m}$ point through a window in the back contact. The spikes in the experimental spectra taken above threshold are scattered stimulated emission. The simulated spectra have a steeper edge on the low energy side than the measured spectra. This is because inhomogeneous broadening due to alloy and QW width fluctuations has not been accounted for, nor have the line shape broadening parameters in the simulation been adjusted to obtain agreement with the measured spectra. Nevertheless, similar trends are observed in the experiment and the simulation. First, the spontaneous emission intensity increases 


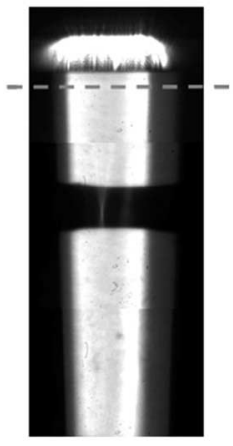

(a)

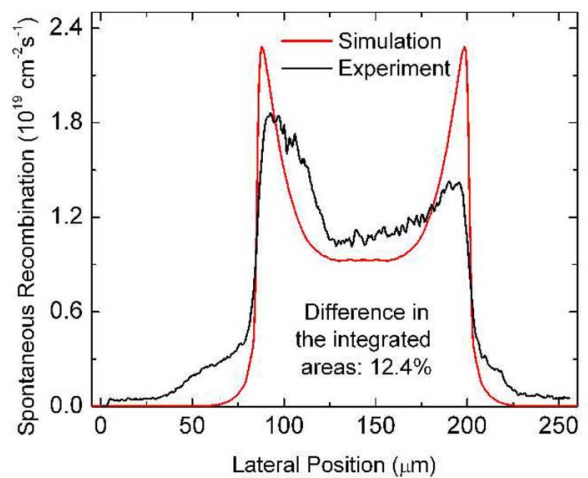

(b)
Fig. 5. (a) Image of the spontaneous emission distribution taken through two windows in the contact near the output facet. (b) Experimental and simulated spontaneous emission profiles at the output facet of a tapered laser [48].

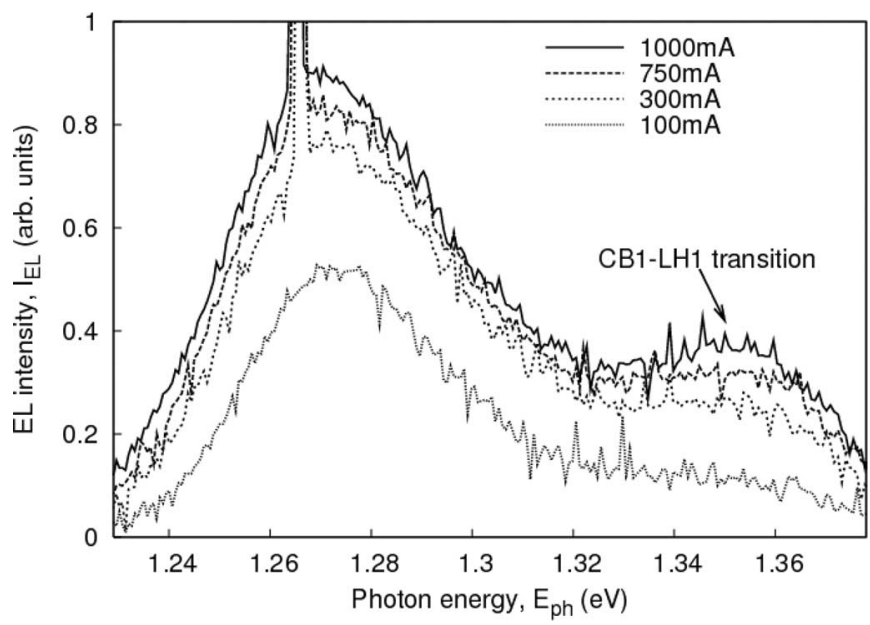

Fig. 6. Experimentally measured spontaneous emission spectra for varying bias taken at the center of the device near the front facet [32].

with bias, due to spectral hole burning. The gain, and hence, carrier density are pinned at the lasing energy (i.e., bottom of the spectral hole). As the bias increases, so does the carrier density around the spectral hole. Second, the CB1-LH1 transition also increases with bias, which can be attributed to carrier heating. This conclusion can be obtained from the simulated spectra, where a larger increase in the CB1-LH1 transition is observed when a nonequilibrium gain model is used, which includes the carrier heating effect.

\section{APPLICATION EXAMPLES}

In this section, application examples are used to demonstrate the role of modeling for the design, simulation, and evaluation of next-generation high-brightness laser diode technologies.

\section{A. Broad-Area Laser Diodes}

Conceptually, the simplest and most obvious way to increase the brightness of a BA-LD is to increase its width, since the far-field and near-field widths are inversely related. The prob-
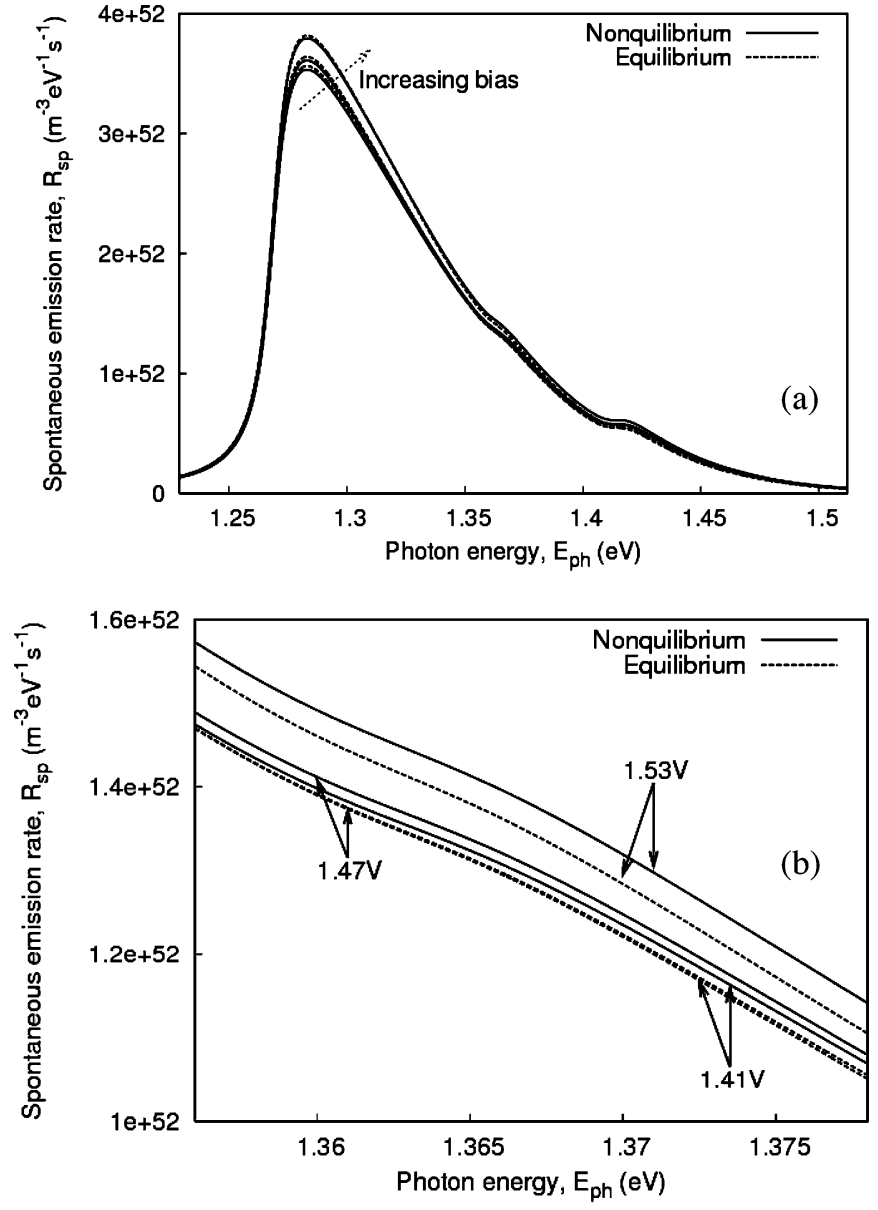

Fig. 7. (a) Simulated spontaneous emission showing nonequilibrium spectral hole burning and carrier heating. (b) Closeup of CB1-LH1 transition [32].

lem is that filaments start to form in the laser. This leads to reduced beam quality, since the narrow filaments cause a large far-field divergence. Furthermore, the number and position of the filaments change both with time and from device to device.

In this study, we investigate the brightness limitations of broad-area lasers by investigating the effect of the resonator geometry on the single-mode operation of a BA-LD. The BALD investigated in this paper is a gain-guided laser with a current stripe defined by deep ion implantation to eliminate current spreading in the cladding layers.

To investigate the operating regime of the BA-LD dominated by the fundamental mode, we employed the Prony method to extract the lateral modes of the BA-LD [49]. The eigenvalues extracted with the Prony method are the round-trip gain/loss of the individual eigenmodes. Thus, if the BA-LD is to operate in a single mode, the fundamental mode should have an eigenvalue equal to unity $\left(\lambda_{0}=1\right)$, while the first-order mode should have an eigenvalue less than unity $\left(\lambda_{1}<1\right)$. If we define the modal discrimination as $\mathrm{MD}=\left|\lambda_{0}\right| /\left|\lambda_{1}\right|$, the BA-LD operates in the single fundamental lateral mode when MD is greater than unity. Thus, to obtain strong single-mode operation, the modal discrimination should be as large as possible. 


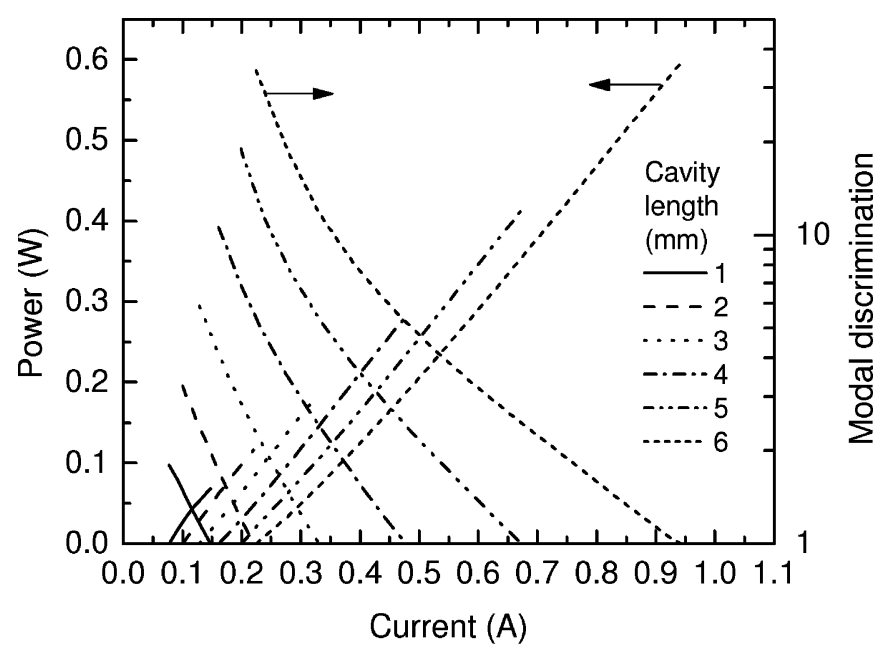

Fig. 8. $L-I$ characteristic and modal discrimination as a function of current bias for cavity lengths $L=1$ to $6 \mathrm{~mm}$. The emitter width is $W=30 \mu \mathrm{m}$ [33].

Before investigating the effect of the other geometrical parameters, the thickness of the vertical waveguide $W_{g}$ needs to be determined. It was shown in [33] that increasing the waveguide thickness allows for higher modal discrimination by reducing the confinement factor, and subsequently, the carrier lensing effect. The carrier lens excites higher order modes in the cavity and promotes the formation of optical filaments. Only a symmetrical vertical structure was considered here with a total vertical waveguide thickness of $2 \mu \mathrm{m}$. To reduce the confinement factor further, it is also possible to employ asymmetric structures, where the QW is positioned closer toward the p-cladding [50].

Having determined the vertical waveguide thickness, the effect of the cavity length is investigated. Fig. 8 shows the variation of modal discrimination and single-mode output power versus bias current. The emitter width is fixed at $30 \mu \mathrm{m}$ and the cavity length is varied from 1 to $6 \mathrm{~mm}$. The longer the cavity length, the higher the modal discrimination is at threshold. Also, as the bias current is increased, the modal discrimination decreases less rapidly for the longer cavity BA-LDs. This can be understood in terms of the round-trip loss of a particular mode $n$ given by $r_{f} r_{b} \exp \left(-\alpha_{n} L\right)$, where $r_{f}$ and $r_{b}$ are the facet reflectances, $\alpha_{n}$ is the net modal loss, and $L$ is the cavity length. The roundtrip loss of the fundamental mode is unity (fixed by the lasing condition), so the modal discrimination increases with cavity length as the loss of the first-order mode increases.

Next, the effect of emitter width is investigated. The cavity length is fixed at $2 \mathrm{~mm}$ and the emitter width is varied from 14 to $28 \mu \mathrm{m}$ (Fig. 9). For narrower emitter widths, the modal discrimination is higher at threshold and decreases less rapidly as a function of bias current. For an emitter width of $14 \mu \mathrm{m}$, the modal discrimination is greater than unity for powers up to $\sim 2 \mathrm{~W}$. This maximum single-mode output power is equivalent to a facet load of $\sim 15 \mathrm{MW} / \mathrm{cm}^{2}$, which is close to the reported catastrophic optical mirror damage (COMD) values of 18-19 MW/ $/ \mathrm{cm}^{2}$ [51]. In addition, since thermal effects and spontaneous emission coupling were not included in these simulations, this represents an upper limit on the maximum bright-

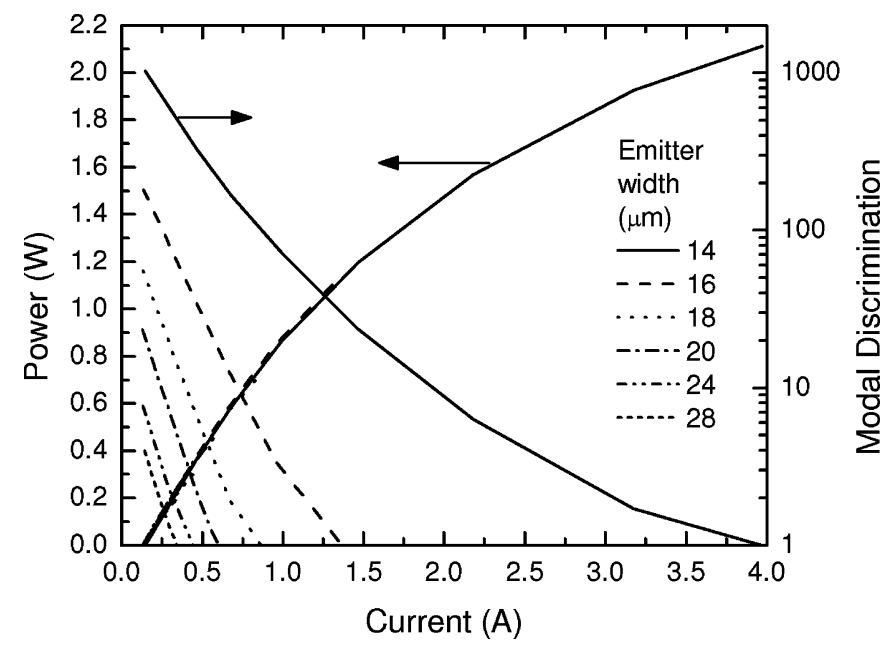

Fig. 9. $L-I$ characteristic and modal discrimination as a function of current for emitter widths $W=14$ to $28 \mu \mathrm{m}$. The cavity length is fixed at $L=2 \mathrm{~mm}[33]$.

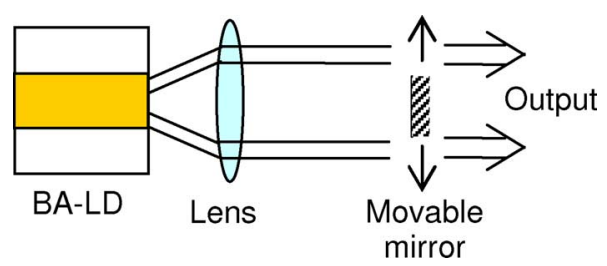

Fig. 10. Schematic diagram of an asymmetric feedback external cavity laser.

ness that can be achieved by a BA-LD. By comparison, tapered lasers (discussed in Section III-C and III-D) have achieved nearly diffraction-limited operation for powers up to $12 \mathrm{~W}$ [2]. Thus, although single-emitter broad-area lasers can produce high output powers, their ultimate brightness is limited by the lack of strong modal discrimination for wide emitters and is restricted by COMD for narrow emitters.

\section{B. Asymmetric Feedback External Cavity Laser}

In this section, an external cavity laser with asymmetric feedback is investigated to improve the modal discrimination and beam quality of a BA-LD. The external cavity laser configuration is shown in Fig. 10. The BA-LD has an emitter width of $100 \mu \mathrm{m}$, a cavity length of $1.5 \mathrm{~mm}$, and an emission wavelength of $975 \mathrm{~nm}$. Details of the epitaxial structure are given in [52]. The front and back facets are antireflection (AR) and high reflection (HR) coated, respectively. A lens with a focal length of $75 \mathrm{~mm}$ is placed at the focal plane to collimate the laser beam along the slow axis. A 0.9 -mm-wide mirror is placed in a far-field plane located $75 \mathrm{~mm}$ from the lens for selective spatial feedback. The 1.5-D isothermal unipolar laser model is used to simulate the BA-LD and is coupled to an external optics module, which models the free-space propagation of the optical beam by solving the Fresnel diffraction equations.

A stable operating condition is found when the mirror stripe is positioned $5.85 \mathrm{~mm}$ from the center. The near-field pattern in Fig. 11(a) has an asymmetric shape, in agreement with 

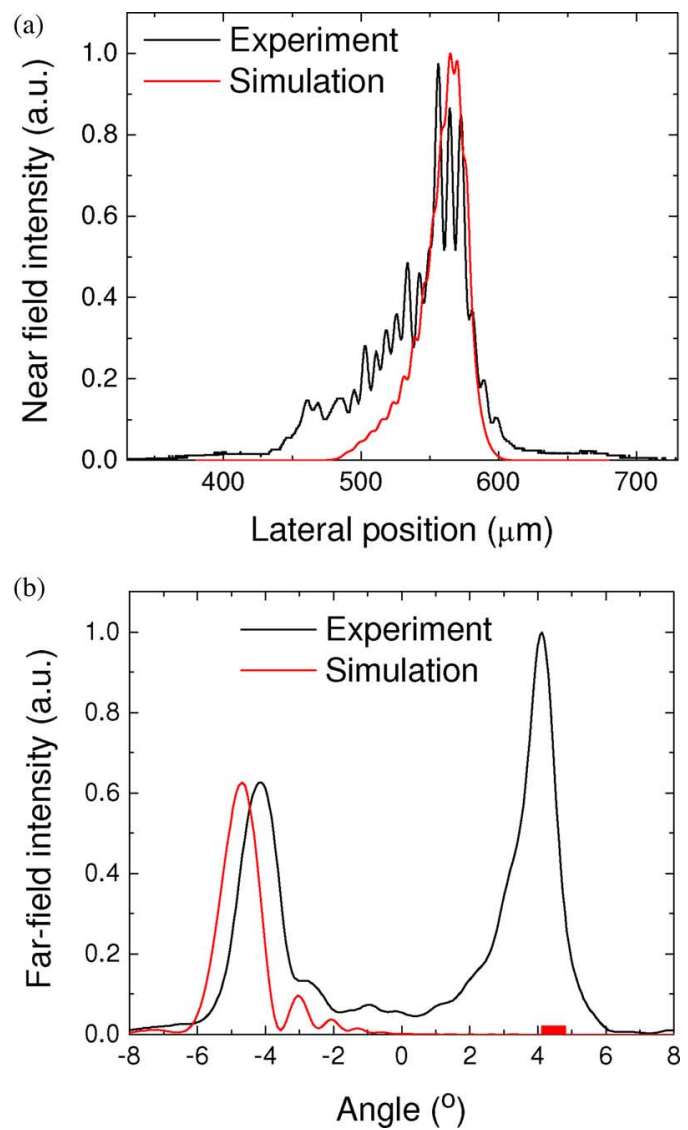

Fig. 11. Converged (a) near-field and (b) far-field patterns of an asymmetric feedback external cavity laser. The mirror width and position used in the simulation are indicated by the stripe in (b).

experiment. The far-field pattern in Fig. 11(b) consists of a narrow main lobe with a full-width at half-maximum (FWHM) of $1.26^{\circ}$, which is offset from the center by $\sim 4.69^{\circ}$. The experimental far-field profile shows a double lobed feature with both a significant output lobe (left) and feedback lobe (right). Such a behavior is uncommon for this asymmetric feedback technique. Usually, a strong and narrow output lobe is observed in the far field [53], in agreement with the simulation, and the feedback lobe is strongly suppressed. The peculiar behavior of this particular laser is due to degradation observed on the front facet AR coating. Nevertheless, the position and width of the experimental lobe coincide well with the simulated profile.

Fig. 12 shows the carrier density (at the front facet) and farfield intensity distributions for different output powers. With increasing power, larger spatial hole burning occurs at the output facet on the opposite side of the feedback mirror. A slight movement of the peak position of the far-field lobe away from the optical axis is seen with increasing power. This is due to spatial hole burning and carrier lensing, which result in a slightly modified waveguiding behavior in the BA-LD.

The simulated $M^{2}$ value using the second moment definition (ISO Standard 11146) is only 2.62, which is small compared to that of a typical BA-LD $\left(M^{2} \approx 30\right)$ [9]. This dramatic improvement is attributed to the increased modal discrimination provided by the mirror stripe, which provides selective feedback
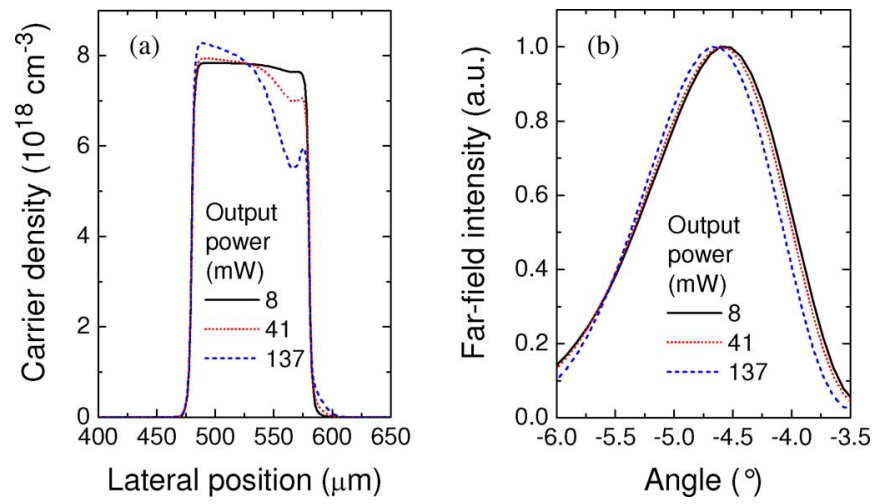

Fig. 12. (a) Carrier density distribution at the output facet. (b) Far-field patterns for different output powers.

to a specific lateral mode. The value of $M^{2} \sim 2.6$ is outstanding for a BA-LD, but many applications require even better beam quality. This can be achieved with tapered lasers, which use a spatial filter to improve the modal discrimination.

\section{Tapered Laser}

Tapered lasers are currently the most popular high-brightness laser diodes. Their superior performance is due to several factors inherent in their design. First, the straight RW section serves as a modal filter, which discriminates against higher order modes. Next, a single lateral mode is allowed to expand adiabatically in the tapered section, minimizing spatial hole burning and filamentation. Finally, its complexity is relatively low compared to other high-brightness lasers, making it suitable for low-cost manufacturing with a high process yield and reliable operation.

Tapered lasers demonstrate clear performance advantages, but filamentation can still be an issue if they are not designed properly. For instance, the RW section geometry must be designed to support a single lateral mode. Figs. 13 and 14 show the 2.5-D spectral simulation of a 5- $\mu$ m-wide RW laser, which just supports two lateral modes. Although the central mode prefers the fundamental lateral mode, the modes at the edges of the emission spectrum clearly show mode beating between the first and second lateral modes. (From the gain profile, it is clear that this is mode beating and not gain or index guiding.) The laser is able to efficiently use the spatial gain distribution in this way, but the result is not good for the modal filtering properties of the RW--particularly as the backward wave from the taper will excite both modes. A narrower RW is needed to suppress the higher order transverse mode, or the loss of the higher order transverse mode must be increased to improve the modal discrimination. Furthermore, to achieve good modal filtering, the taper angle should match the free diffraction angle of the mode launched into the tapered section, and the epitaxial structure should have a low modal gain to reduce carrier lensing effects.

Along with the RW design, the front facet reflectivity and beam spoiler also affect the modal filtering performance of the tapered laser. From earlier investigations on a 2-mm-long 


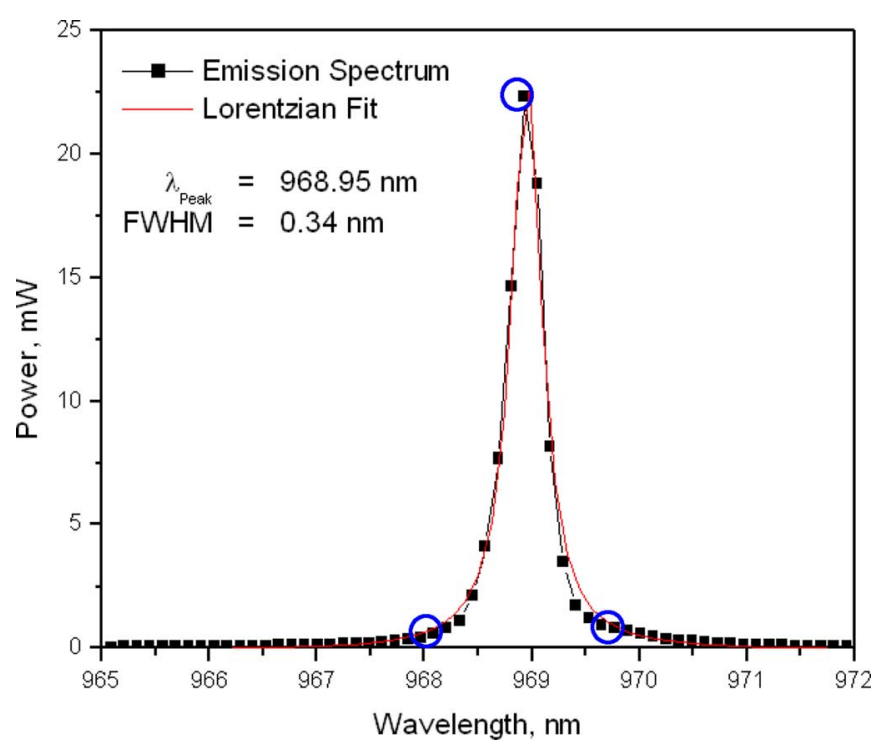

Fig. 13. Simulated emission spectrum of a 5- $\mu$ m-wide index-guided RW laser. The circled points correspond to the fields shown in Fig. 14.

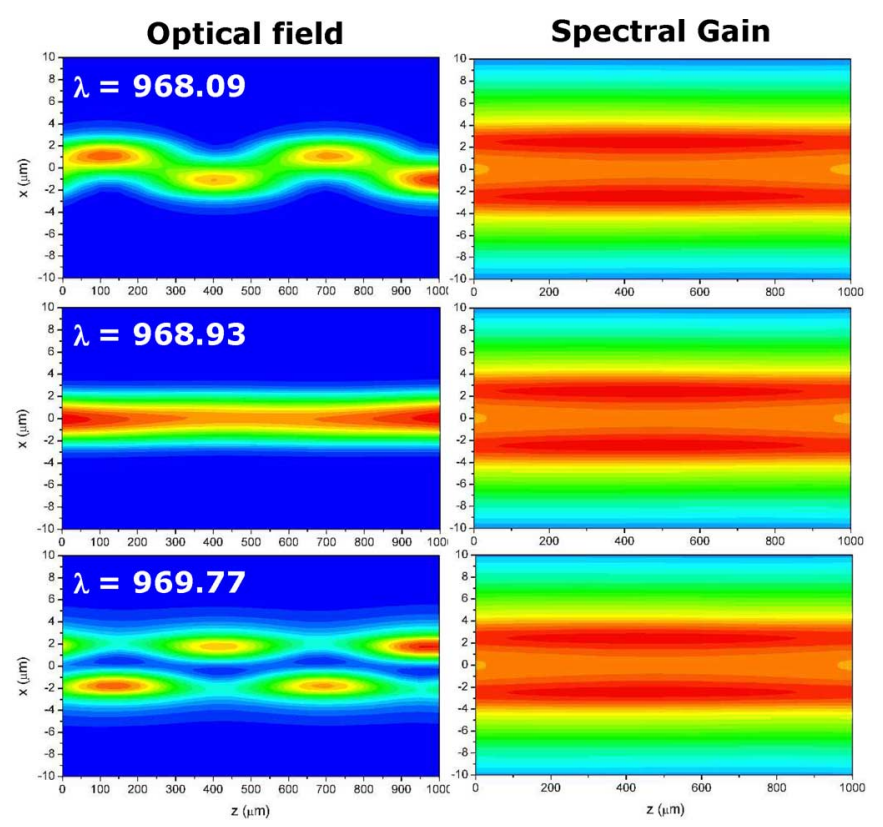

Fig. 14. Spectral field (left) and gain (right) distributions of a 5- $\mu \mathrm{m}$-wide index-guided 970-nm RW laser at wavelengths of 968.09 (top), 968.93 (middle), and $969.77 \mathrm{~nm}$ (bottom).

$4^{\circ}$ gain-guided tapered laser (using a different monochromatic 2.5-D laser model [30]), it was shown that a front facet reflectivity $R_{f}=1 \%$ produced filamentation in the near-field profile. This filamentation is caused by optical pumping of the regions adjacent to the RW, which become transparent and reduce the modal filtering efficiency of the RW section. This resulted in small side lobes in the forward propagating wave in the RW section, which seed the filamentation process and create a multilobed near-field pattern. When $R_{f}$ is reduced to $0.1 \%$, the optical bleaching of the RW section is avoided. This improves

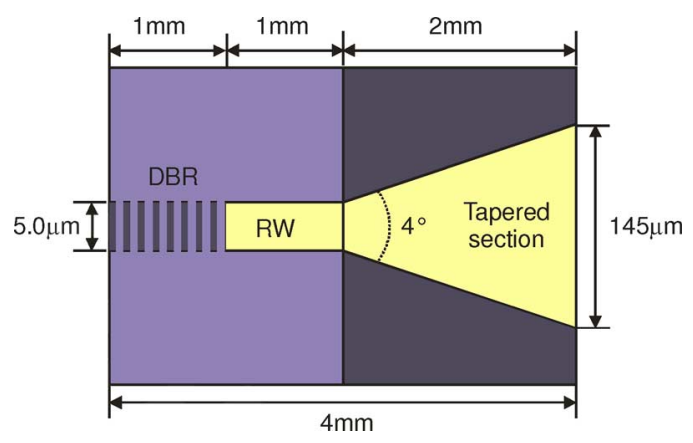

Fig. 15. Schematic diagram of the DBR tapered laser.

the modal filtering in the RW and eliminates the filamentation in the taper. Beam spoilers were also shown to prevent the optical bleaching of the regions adjacent to the RW. Even with the original front facet reflectivity of $1 \%$, a $6-\mu \mathrm{m}$ aperture placed $100 \mu \mathrm{m}$ from the rear facet filtered the high-order modes out of the relatively intense backward wave and prevented filamentation and beam quality degradation.

\section{Multisection Tapered Laser}

There is growing interest in using tapered lasers in display and optical wireless applications due to their good beam quality and high output power. These applications require a high modulation efficiency $(d P / d I)$ to minimize the cost and complexity of the laser driver. This can be achieved through the use of separate contacts for the RW and tapered amplifier sections. By using a small modulation bias on the RW and keeping the tapered section bias fixed, higher modulation bandwidths and efficiency can be achieved. To satisfy the targeted applications, these lasers should have a stable beam quality and astigmatism for all bias conditions, since the lenses that collimate and focus the output beam of the laser are designed for fixed beam parameters.

Here, we investigate a 1060-nm DBR tapered laser intended for generating $530 \mathrm{~nm}$ light by second harmonic generation (SHG) for laser displays [41]. At the end of the RW, the DBR tapered laser includes a passive DBR section to fix the emission wavelength, as shown in Fig. 15. High spectral brightness and stability (i.e., a narrow and stable spectral linewidth) is required by the nonlinear crystal for SHG. Since the DBR section locks the emission wavelength, we have only simulated a single wavelength fixed at $1060 \mathrm{~nm}$. The lasing wavelength was measured to be stable with RW current within 130 pm [41]. The good wavelength stability with respect to current is due to efficient thermal management and the small temperature and index change in the passive DBR section. The index-guided RW has an index step of $\sim 1.5 \times 10^{-3}$, while the taper is gain guided with a shallow implant for electrical isolation.

The DBR section is passive (unpumped), so it has been represented by a fixed reflectivity at the end of the RW. The reflectivity of the sixth-order DBR grating of DBR RW lasers was found to be around $31 \%$ [41]. We used a top-hat profile (in the lateral direction) for the reflectivity with a value of $31 \%$ for the region within the RW and a value of $0.01 \%$ outside the RW. The low 


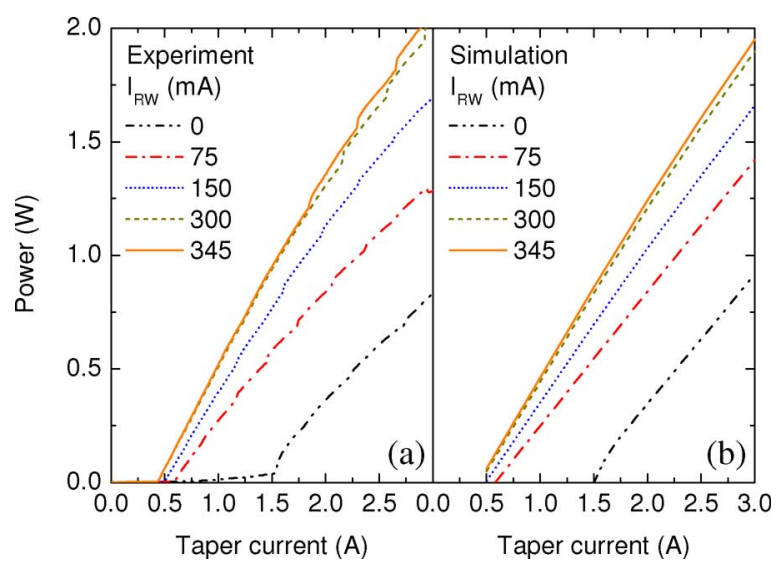

Fig. 16. (a) Experimental and (b) simulated light-current characteristics.

reflectivity of the region outside the RW was used, since any backward traveling light is strongly absorbed in the unpumped region in the DBR section and very little light is reflected back from the AR-coated back facet $(<0.1 \%)$. The front facet was AR-coated to $1 \%$.

The DBR tapered laser is simulated using the 2.5-D monochromatic laser model, employing the material parameters determined in Section II-B. Fig. 16 compares (a) the experimental and (b) simulated output power versus taper current characteristics of a split-contact 1060-nm DBR tapered laser. Good agreement is obtained over the range of RW and taper currents investigated. The saturation of the output power with increasing RW current is reproduced in the simulations and is due to the depletion of carriers in the tapered amplifier with increasing optical injection from the RW.

The photon density distributions for the combined forward and backward waves are plotted in Fig. 17 for RW currents of (a) $0 \mathrm{~mA}$ and (b) $345 \mathrm{~mA}$ at a fixed taper current of $1.6 \mathrm{~A}$. The output powers are 100 and $938 \mathrm{~mW}$, respectively. For these conditions, the simulated modulation efficiency is $2.43 \mathrm{~W} / \mathrm{A}$, which is slightly smaller than experiment (2.6 W/A). The simulated extinction ratio is 9.4, which is bigger than the experimental value of 7.7. (The extinction ratio is expected to be squared for the frequency-doubled green output.) No filamentation is observed for either RW current, due to the good modal filtering by the passive DBR and low AR coating on the back facet. The lack of filamentation is also partly due to the use of a taper angle that matches the free diffraction angle of the beam from the RW. A larger increase in photon density toward the front facet in the tapered section is observed for $I_{\mathrm{RW}}=0 \mathrm{~mA}$ compared to $I_{\mathrm{RW}}=345 \mathrm{~mA}$, which demonstrates the saturation of the tapered amplifier as the RW current is increased.

The experimental and simulated far-field patterns are shown in Fig. 18. There are discrepancies between the simulated and measured far-field patterns, especially at the larger RW currents. The lack of perfect agreement is probably because the simulations were performed with calculated carrier-induced changes in the refractive index spectra, which do not include index changes in the waveguide core or cladding. Furthermore, index changes near the emission wavelength are very sensitive to temperature
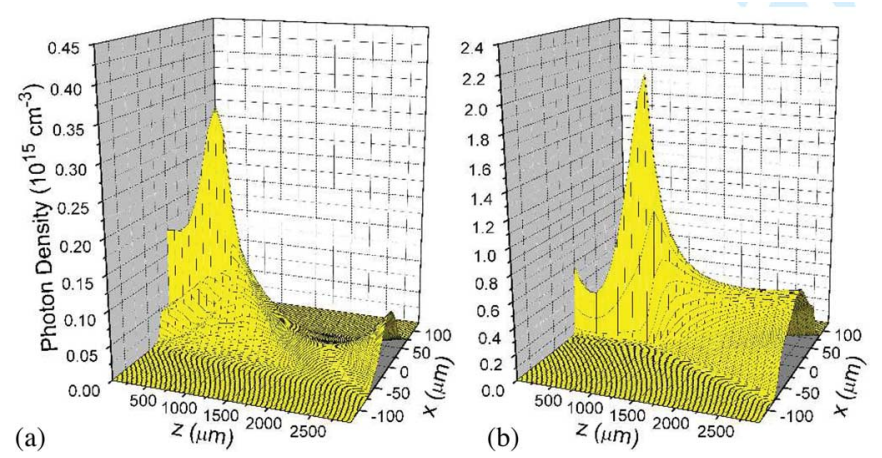

Fig. 17. Simulated photon density distributions (forward and backward waves) at a fixed taper current of $1.6 \mathrm{~A}$ and RW currents of (a) $0 \mathrm{~mA}$ and (b) $345 \mathrm{~mA}$.
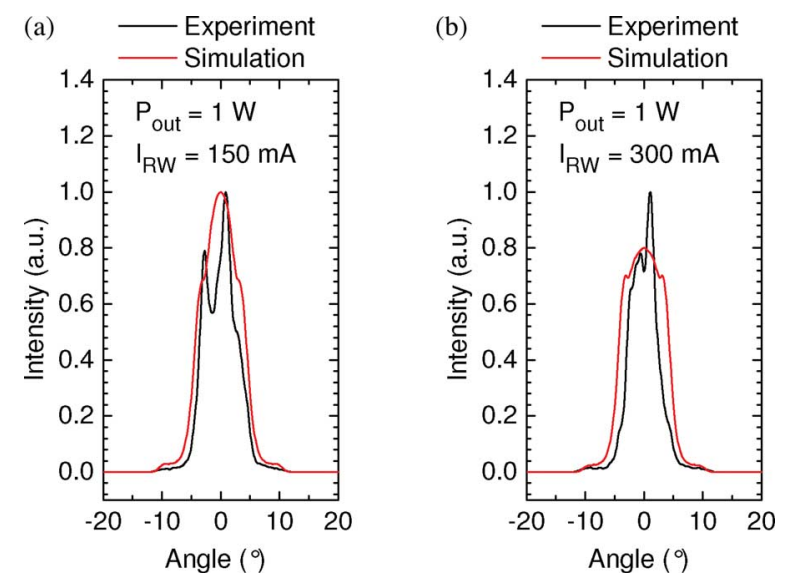

Fig. 18. Measured and simulated far-field profiles for a fixed output power of $1 \mathrm{~W}$ and RW currents of (a) $150 \mathrm{~mA}$ and (b) $300 \mathrm{~mA}$.

changes. The thermal conductivity of the package was not independently measured, since this is usually extracted from changes in the emission wavelength above threshold. (As the emission wavelength is fixed by the DBR, this does not work here.)

Fig. 19 shows a plot of the beam quality factor and the astigmatism versus $I_{\mathrm{RW}}$ at a fixed output power of $1 \mathrm{~W}$. The $M^{2}$ factor (determined using the $1 / e^{2}$ level) is in reasonable agreement with experiment and stays at a fairly constant value of $\sim 1.2$. Fig. 19 shows that the simulated astigmatism of the beam stays fairly stable versus $I_{\mathrm{RW}}$, while the experimental data increase slightly with $I_{\mathrm{RW}}$. The agreement with experiment seems reasonable, but is expected to improve once the spectral index change is experimentally validated and calibrated.

The stability of the $M^{2}$ factor and the astigmatism with respect to $I_{\mathrm{RW}}$ is due to the use of a thick vertical waveguide $(4.8 \mu \mathrm{m})$, since the carrier-induced index depression is reduced by the small confinement factor. The exceptional beam quality and modulation efficiency are, in part, due to the use of an AR coating instead of an HR coating on the rear facet and the presence of the passive DBR section. This improves the filtering of the higher order modes in the RW section-an approach not previously considered. 


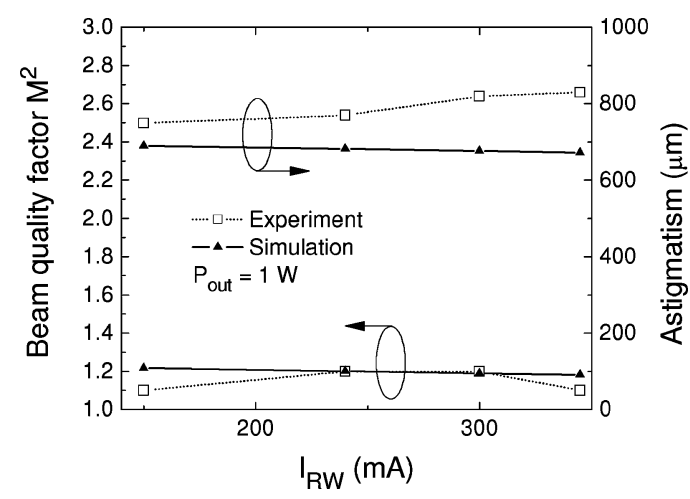

Fig. 19. Beam quality factor and astigmatism versus RW current. The output power is fixed at $1 \mathrm{~W}$.

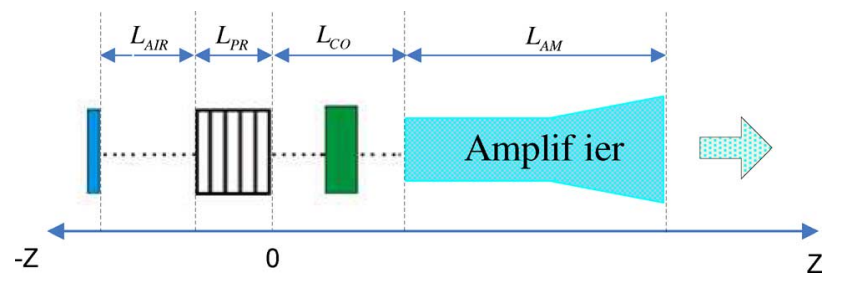

Fig. 20. Schematic diagram of the self-organizing external cavity laser. $L_{\mathrm{AIR}}$ : length of air gap; $L_{\mathrm{PR}}$ : length of the PR crystal; $L_{\mathrm{CO}}$ : length of the collimating optics section; $L_{\mathrm{AM}}$ : length of the tapered amplifier.

\section{E. Self-Organizing External Cavity Laser}

In laser technology, photorefractive (PR) crystals are typically used in two situations: 1) in tunable external cavity semiconductor lasers, where the PR crystal relaxes the alignment tolerances and 2) in self-organizing external cavity lasers, where it is used to make an adaptive Fabry-Perot mirror to enhance the side mode suppression ratio.

The schematic diagram of a self-organizing external cavity laser is shown in Fig. 20, where a PR crystal is placed between a high reflectivity mirror and the rear facet of the high-power tapered amplifier. The purpose of assembling a laser cavity in this particular configuration is to deliver a beam with high spectral and spatial brightness. By using a PR crystal instead of a fixed DBR, the laser cavity self-adapts to its operating conditions and ensures maximum efficiency.

The model of this self-organizing external cavity laser consists of a PR crystal model, which relies on a phenomenological plane wave approach [54], and the 1.5-D spectral laser model. (A 1-D unipolar, isothermal electrical model is used for numerical efficiency.) In the simulation, the PR crystal and the rear mirror are treated as a single adaptive Fabry-Perot filter, which provides wavelength-dependent feedback to the tapered amplifier. The filter reflectivity was calculated by solving a set of coupled wave equations [54]. The main approximation of the model is that the mode with the largest power writes the grating. The other longitudinal modes are scattered from this grating.

The simulation of the self-organizing high-power external cavity laser is performed in three stages. Initially, the resonant

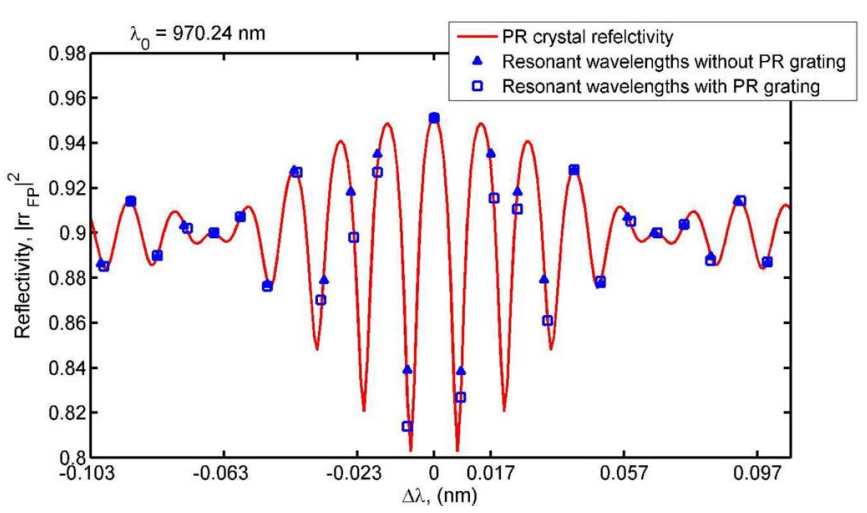

Fig. 21. Dependence of the Fabry-Perot filter reflectivity on wavelength [55].

wavelength of the main longitudinal mode is determined by the spectral simulation of the free running laser, i.e., while neglecting the formation of the grating in the PR crystal. This simplification is justified because there is no phase shift (and hence also no resonant wavelength shift) introduced by the PR grating for the main mode. A 1-D simulation cannot be used for this purpose, since the energy of the gain maximum depends on the carrier density distribution in the tapered amplifier, which depends strongly on position.

Once the wavelength of the main longitudinal mode is determined, the reflectivity of the PR crystal grating is obtained, along with the values of the resonant wavelengths of all the side modes. The calculated values of the resonant wavelength for the cavity modes are shown in Fig. 21. The resonant wavelengths were calculated with and without the phase shift introduced by the grating. These results confirm that the recorded PR crystal grating has a significant impact on the position and the reflectivity of the cavity modes. Without the induced grating, the reflectivity of all the modes would be equal to that of the mirror.

After the wavelengths of all the cavity modes and the corresponding reflectivities are known, the full spectral simulation of the laser is performed. Fig. 22 compares the output power spectra, calculated for a bias current of $0.32 \mathrm{~A}$, for an external cavity laser with and without PR crystal. The simulations show that the PR crystal produces single longitudinal mode operation. The spectral linewidth is less than $18 \mathrm{pm}$, since the longitudinal mode spacing is only $9 \mathrm{pm}$. Single-mode operation was also found experimentally, where the instantaneous linewidth was measured to be $\sim 80 \mathrm{MHz}(<0.3 \mathrm{pm})$ and is stable up to $<2 \mathrm{pm}$. The simulated side mode suppression ratio is high $(>50 \mathrm{~dB})$, as seen experimentally $(>30 \mathrm{~dB}$ limited by the spectrum analyzer).

Previously, self-organized cavity laser diodes have only been modeled with 0-D rate equations. The results reported here demonstrate the 1.5-D spectral simulation of the spectral brightness of these devices. The next challenge is to make the wavelength selection respond to changes in the operating conditions. This requires a more advanced model for the PR crystal. 


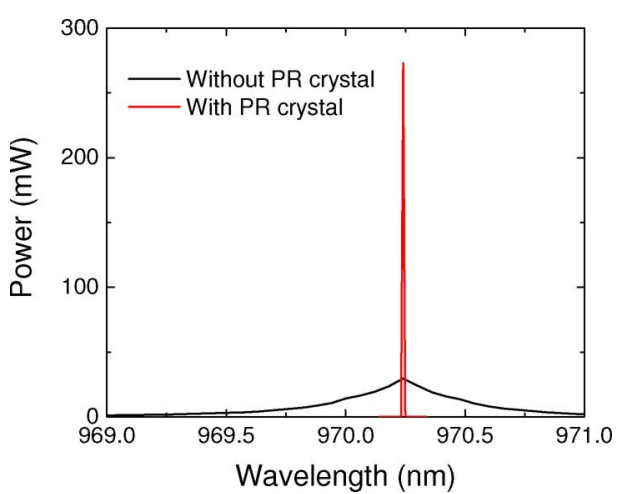

Fig. 22. Calculated output power spectrum for external cavity laser with and without the PR crystal.

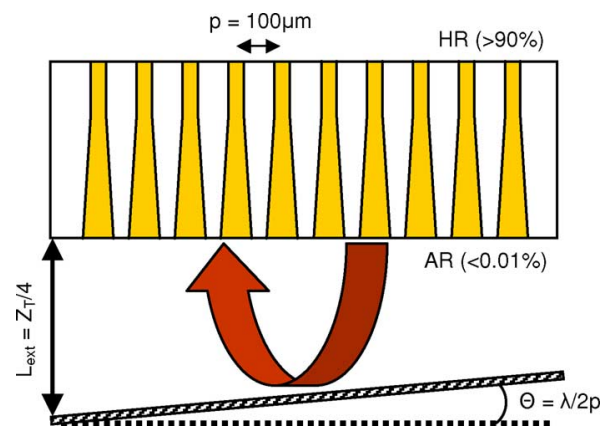

Fig. 23. Schematic diagram of the external Talbot cavity laser.

\section{F. External Talbot Cavity Laser}

Phase-locking of an array of emitters has received great attention for achieving highly coherent emission with high output power. Phase-locking can be induced using the Talbot effect [17], where the out-of-phase mode is self-imaged and the in-phase mode is imaged with a lateral shift of $p / 2$ (where $p$ is the emitter pitch) after traveling half the Talbot distance $\left(Z_{T}=2 p^{2} / \lambda\right)$. By placing a mirror in an external cavity at a distance $Z_{T} / 4$ with a tilt of $\lambda / 2 p$ (Fig. 23), the in-phase mode is self-imaged while the out-of-phase mode is laterally displaced by $p / 2$. This increases the modal discrimination between the in-phase and out-of-phase modes, thus phase-locking the emitters in the in-phase mode.

The array studied here consists of ten narrow index-guided tapered lasers emitting at $975 \mathrm{~nm}$. Further details of the tapered laser and epitaxial structure can be found in [52] and [56].

For the simulations, the 1.5-D isothermal unipolar laser model was coupled to a free-space propagation model (based on the Fresnel diffraction equations) for the external cavity [57]. The reflectivity of the tilted output mirror was $40 \%$. The operation of the Talbot cavity was confirmed by investigating the effect of the tilt of the mirror. With an untilted mirror, the laser oscillates in the out-of-phase mode, but with a tilt angle of $\lambda / 2 p$, the laser operates in the in-phase mode. This confirms the strong modal discrimination of the Talbot cavity.

The front facet near-field distribution (intensity and phase) for the in-phase and out-of-phase modes are shown in Fig. 24. Comprehensive modeling of the beam propagation in the whole (a)

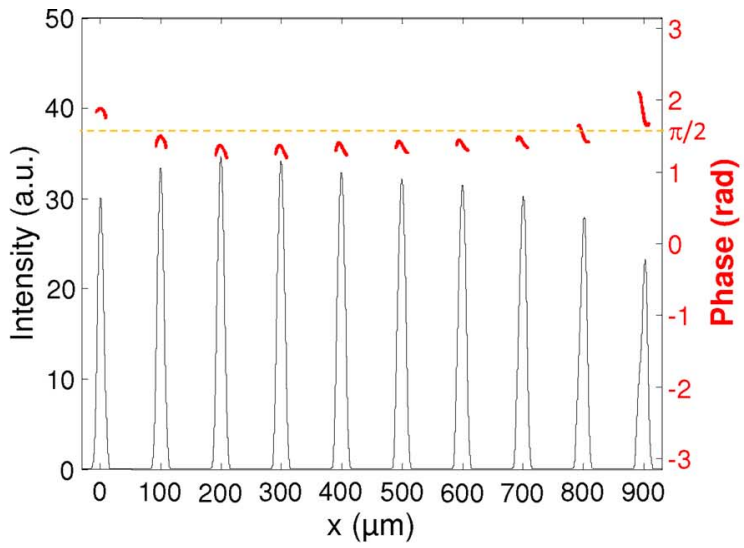

(b)

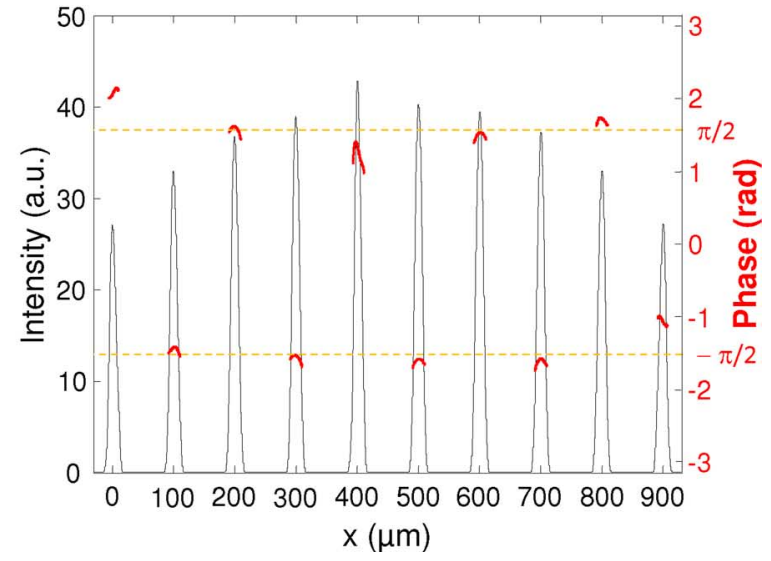

Fig. 24. Field intensity and phase at the front facet for the (a) in-phase and (b) out-of-phase mode operation at an operating current of $3.88 \mathrm{~A}$.

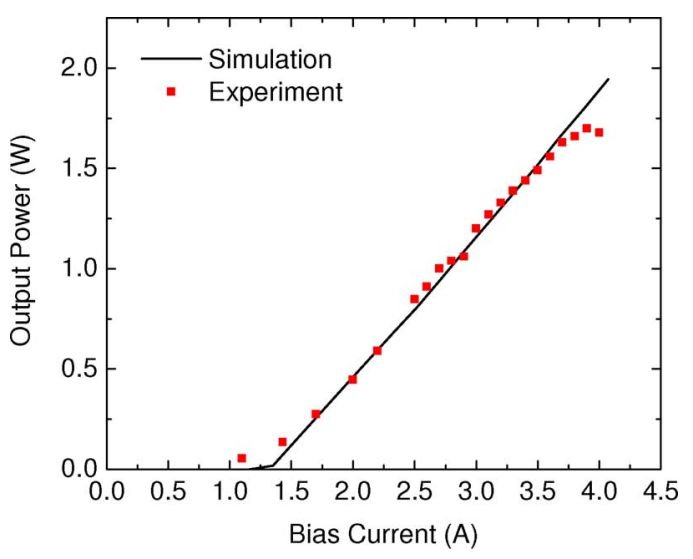

Fig. 25. Experimental versus simulated $L-I$ characteristic.

cavity predicts some interesting features of the spatial modes under external cavity operation and leads to better understanding of the laser array behavior. The asymmetry of the intensity profile of the in-phase mode is a consequence of the tilt of the mirror. In both cases, the phase distributions show the expected in-phase and out-of-phase behavior.

Fig. 25 shows that the simulated $L-I$ characteristics for the in-phase supermode operation of the laser bar agree well with experiment. The slight discrepancies are probably due to current spreading and self-heating effects in the experiments. 


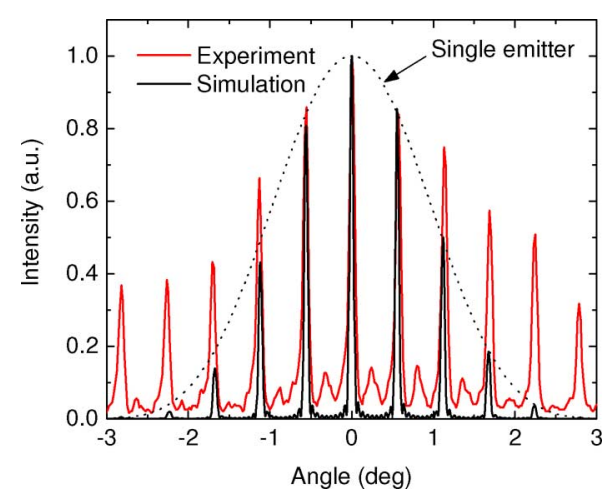

Fig. 26. Far-field profile for the in-phase mode operation at a current of $3 \mathrm{~A}$.

In Fig. 26, the simulated far-field profile for lasing of the in-phase mode shows interference peaks, which agree with experiment. The simulated and measured interference peaks have a width of $0.07^{\circ}$. The envelope of the simulated profile has a Gaussian shape, corresponding to the output of a single emitter. The experimental far-field envelope is wider due to the presence of multimode emission from the tapered emitters.

These results demonstrate the model's ability to reproduce the experimental characteristics and provide insight into the field distribution in the array. Further work will include the detailed investigations of the modal discrimination behavior of the laser and the use of the 2.5-D model to accurately investigate the impact of carrier and thermal lensing effects.

\section{CONCLUSION}

To satisfy increasing application demands and enter new markets, next-generation high-brightness laser diodes require not only good beam quality but also high spectral brightness and added functionality (e.g., direct modulation). Several nextgeneration high-brightness laser diodes were considered, including the asymmetric feedback broad-area laser, the multisection tapered laser, the self-organizing cavity laser, and the external Talbot cavity laser.

Using the Prony method, it was shown that the modal discrimination of BA-LD improves as the cavity width decreases and the cavity length and waveguide thickness increase. To achieve high power levels, the devices must be quite long (up to $6 \mathrm{~mm}$ ) and the width of the cavity must be narrow. This results in a high facet load of $>100 \mathrm{~mW} / \mu \mathrm{m}$. Using asymmetric feedback to increase the modal discrimination dramatically improves the beam quality and reduces $M^{2}$ from $\sim 30$ to $\sim 2.6$, without strong limitations on cavity length or width. Nevertheless, their performance falls short of that of recent tapered lasers. Still, asymmetric feedback may play a role in external cavity approaches for the phase coupling of high-power BA-LD arrays.

Methods to improve the brightness of tapered lasers were demonstrated, including optimizing the RW design to improve the modal discrimination, reducing the front facet AR coating, and using a beam spoiler. Through proper calibration, the laser simulator is able to reproduce the experimental operating characteristic of the multisection 1060-nm DBR tapered laser with good accuracy. The DBR tapered laser has stable spectrum, $M^{2}$, and astigmatism versus RW current, which are important for efficient frequency doubling through a nonlinear crystal. The rear reflector, comprised of a passive DBR with an AR-coated back facet, improved the modulation efficiency of the device.

The spatial and spectral brightness of a tapered selforganizing cavity laser was simulated for the first time. This approach provides a mechanism for improving the spectral brightness of lasers without incurring strict alignment constraints. Finally, we demonstrated the simulation of phase-locking in an array of independent tapered lasers through the use of an external Talbot cavity. The in-phase and out-of-phase modes can be selected by changing the tilt of the external mirror

In summary, the complexity of the devices and increasing range of exacting performance specifications make the use of accurate simulation tools essential for the design and optimization of next-generation high-brightness laser diodes and systems. The examples presented demonstrate the need for laser models that include beam propagation and spectral effects. The beam propagation model is needed to reproduce the evolution with temperature and current of the near- and far-field distributions, $M^{2}$, and the astigmatism. It is also needed for diffraction effects, which are important for the phase coupling of emitters. Spectral models are needed to evaluate the spectral brightness of a laser and the dependence of the lasing wavelength on temperature and current. Finally, models that are able to handle more general structures are needed to simulate a wider range of devices such as multicontact lasers, external cavity lasers, and phase-coupled arrays to address new and emerging applications.

\section{ACKNOWLEDGMENT}

The authors gratefully acknowledge L. Borruel and I. Esquivias for their contribution to the work on tapered lasers in Section III-C and T. M. Benson for useful discussions about optical modeling.

\section{REFERENCES}

[1] R. Diehl, Ed., High-Power Diode Lasers: Fundamentals, Technology, Applications. Berlin, Germany: Springer-Verlag, 2000.

[2] B. Sumpf, Ferdinand-Braun-Institut für Höchstfrequenztechnik, Berlin, private communication, Oct. 2008.

[3] J. R. Marciante and G. P. Agrawal, "Spatio-temporal characteristics of filamentation in broad-area semiconductor lasers: Experimental results," IEEE Photon. Technol. Lett., vol. 10, no. 1, pp. 54-56, Jan. 1998.

[4] Z. Bao, R. K. DeFreez, P. D. Carleson, C. Largent, C. Moeller, and G. C. Dente, "Spatio-spectral characteristics of a high power, high brightness CW InGaAs/AlGaAs unstable resonator semiconductor laser," Electron. Lett., vol. 29, no. 18, pp. 1597-1599, 1993.

[5] L. M. Tilton, G. C. Dente, A. H. Paxton, J. Cser, R. K. DeFreez, C. E. Moeller, and D. Depatie, "High power, nearly diffraction-limited output from a semiconductor laser with an unstable resonator," IEEE J. Quantum Electron., vol. 27, no. 9, pp. 2098-2108, Sep. 1991.

[6] R. Parke, D. F. Welch, A. Hardy, R. Lang, D. Mehuys, S. Obrien, K. Dzurko, and D. Scifres, "2.0 W CW, diffraction-limited operation of a monolithically integrated master oscillator power-amplifier," IEEE Photon. Technol. Lett., vol. 5, no. 3, pp. 297-300, May 1993.

[7] E. S. Kintzer, J. N. Walpole, S. R. Chinn, C. A. Wang, and L. J. Missaggia, "High power, strained-layer amplifiers and lasers with tapered gain regions," IEEE Photon. Technol. Lett., vol. 5, no. 6, pp. 605-608, Jun. 1993. 
[8] D. Masanotti and F. Causa, "Optical guiding properties of high-brightness parabolic bow-tie laser arrays," IEEE J. Quantum Electron., vol. 41, no. 7, pp. 909-916, Jul. 2005.

[9] R. J. Lang, K. M. Dzurko, A. Hardy, S. Demars, A. Schoenfelder, and D. F. Welch, "Theory of grating-confined broad-area lasers," IEEE J. Quantum Electron., vol. 34, no. 11, pp. 2197-2210, Nov. 1998.

[10] K. Paschke, R. Güther, J. Fricke, F. Bugge, G. Erbert, and G. Tränkle, "High power and high spectral brightness in $1060 \mathrm{~nm} \alpha$-DFB lasers with long resonators," Electron. Lett., vol. 39, no. 4, pp. 369-370, 2003.

[11] K. Paschke, A. Bogatov, A. E. Drakin, R. Güther, A. A. Stratonnikov, H. Wenzel, G. Erbert, and G. Tränkle, "Modelling and measurements of the radiative characteristics of high-power $\alpha$-DFB lasers," IEEE J. Sel. Topics Quantum Electron., vol. 9, no. 3, pp. 835-43, May/Jun. 2003.

[12] B. Thestrup, M. Chi, B. Sass, and P. M. Petersen, "High brightness laser source based on polarization coupling of two diode lasers with asymmetric feedback," Appl. Phys. Lett., vol. 82, no. 5, pp. 680-682, 2003.

[13] S. Wolff, A. Rodionov, V. E. Sherstobitov, and H. Fouckhardt, "Fourieroptical transverse mode selection in external-cavity broad-area lasers: Experimental and numerical results," IEEE J. Quantum Electron., vol. 39 , no. 3, pp. 448-458, Mar. 2003.

[14] D. E. Ackley, "Single longitudinal mode operation of high power multiplestripe injection lasers," Appl. Phys. Lett., vol. 42, no. 2, pp. 152-154, 1983.

[15] H. Yang, L. J. Mawst, M. Nesnidal, J. Lopez, A. Bhattacharya, and D. Botez, "10 W near-diffraction-limited peak pulsed power from Al-free, $0.98 \mu \mathrm{m}$-emitting phase-locked antiguided arrays," Electron. Lett., vol. 33, no. 2, pp. 136-137, 1997.

[16] L. J. Mawst, D. Botez, T. J. Roth, W. W. Simmons, G. Peterson, M. Jansen, J. Z. Wilcox, and J. J. Yang, "Phase-locked array of antiguided lasers with monolithic spatial filter," Electron. Lett., vol. 25, no. 5, pp. 365-366, 1989.

[17] J. R. Leger, "Lateral mode control of an AlGaAs laser in a Talbot cavity," Appl. Phys. Lett., vol. 55, no. 4, pp. 334-336, 1989.

[18] F. Camacho, C. J. Hamilton, K. McIlvaney, A. C. Bryce, and J. H. Marsh, "Laser structure for generating high optical power in a single mode waveguide," Electron. Lett., vol. 34, pp. 460-461, Mar. 1998.

[19] R. J. Lang, A. G. Larsson, and J. G. Cody, "Lateral modes of broad area semiconductor lasers: Theory and experiment," IEEE J. Quantum Electron., vol. 27, no. 3, pp. 312-320, Mar. 1991.

[20] Z.-M. Li, K. M. Dzurko, A. Delage, and S. P. McAlister, "A self-consistent two dimensional model of quantum well semiconductor lasers: Optimization of a GRIN-SCH SQW laser structure," IEEE J. Quantum Electron., vol. 28, no. 4, pp. 792-803, Apr. 1992.

[21] M. Grupen and K. Hess, "Simulation of carrier transport and nonlinearities in quantum well laser diodes," IEEE J. Quantum Electron., vol. 34, no. 1, pp. 120-139, Jan. 1998.

[22] M. A. Alam, M. S. Hybertsen, R. K. Smith, and G. A. Baraff, "Simulation of semiconductor quantum well lasers," IEEE Trans. Electron Devices, vol. 47, no. 10, pp. 1917-1925, Oct. 2000.

[23] G. P. Agrawal, "Fast-Fourier-transform based beam-propagation model for stripe-geometry semiconductor-lasers-inclusion of axial effects," $J$. Appl. Phys., vol. 56, pp. 3100-3109, 1984.

[24] M. Mikulla, P. Chazan, A. Schmitt, S. Morgott, A. Wetzel, M. Walther, R. Kiefer, W. Pletschen, J. Braunstein, and G. Weimann, "High-brightness tapered semiconductor laser oscillators and amplifiers with low-modal gain epilayer structures," IEEE Photon. Technol. Lett., vol. 10, no. 5, pp. 654-656, May 1998.

[25] K. A. Williams, R. W. Penty, I. H. White, D. J. Robbins, F. J. Wilson, J. J. Lewandowski, and B. K. Nayar, "Design of high-brightness tapered laser arrays," IEEE J. Sel. Topics Quantum Electron., vol. 5, no. 3, pp. 822831, May/Jun. 1999.

[26] O. Hess, S. W. Koch, and J. V. Moloney, "Filamentation and beam propagation in broad-area semiconductor lasers," IEEE J. Quantum Electron., vol. 31, no. 1, pp. 35-43, Jan. 1995.

[27] J. V. Moloney, R. A. Indik, and C. Z. Nong, "Full space time simulation for high brightness semiconductor lasers," IEEE Photon. Technol. Lett., vol. 9, no. 6, pp. 731-733, Jun. 1997.

[28] B. Witzigmann, A. Witzig, and W. Fichtner, "A multidimensional laser simulator for edge-emitters including quantum carrier capture," IEEE Trans. Electron Devices, vol. 47, no. 10, pp. 1926-2000, Oct. 2000.

[29] U. Bandelow, R. Hünlich, and T. Koprucki, "Simulation of static and dynamic properties of edge-emitting multiple-quantum-well lasers," IEEE J. Sel. Topics Quantum Electron., vol. 9, no. 3, pp. 798-806, May/Jun. 2003.

[30] S. Sujecki, L. Borruel, J. Wykes, P. Moreno, B. Sumpf, P. Sewell, H. Wenzel, T. M. Benson, G. Erbert, I. Esquivias, and E. C. Larkins, "Nonlinear properties of tapered laser cavities," IEEE J. Sel. Topics Quantum Electron., vol. 9, no. 3, pp. 823-834, May/Jun. 2003.

[31] L. Borruel, S. Sujecki, P. Moreno, J. Wykes, M. Krakowski, B. Sumpf, P. Sewell, S.-C. Auzanneau, H. Wenzel, D. Rodríguez, T. M. Benson, E. C. Larkins, and I. Esquivias, "Quasi-3-D simulation of high-brightness tapered lasers," IEEE J. Quantum Electron., vol. 50, no. 5, pp. 463-472, May 2004.

[32] P. J. Bream, J. J. Lim, S. Bull, A. V. Andrianov, S. Sujecki, and E. C. Larkins, "The impact of nonequilibrium gain in a spectral laser diode model," Opt. Quantum Electron., vol. 38, pp. 1019-1027, Sep. 2006.

[33] J. J. Lim, T. M. Benson, and E. C. Larkins, "Design of wide-emitter single-mode laser diodes," IEEE J. Quantum Electron., vol. 41, no. 4, pp. 506-516, Apr. 2005.

[34] G. R. Hadley, "Multistep method for wide-angle beam propagation," Opt. Lett., vol. 17, pp. 1743-1745, 1992.

[35] R. Mackenzie, J. J. Lim, S. Bull, S. Sujecki, and E. C. Larkins, "Inclusion of thermal boundary resistance in the simulation of high-power $980 \mathrm{~nm}$ ridge waveguide lasers," Opt. Quantum Electron., vol. 40, no. 5/6, pp. 373-377, Apr. 2008.

[36] R. Mackenzie, J. J. Lim, S. Bull, S. Sujecki, A. J. Kent, and E. C. Larkins, "The impact of hot-phonons on the performance of dilute nitride edgeemitting quantum well lasers," J. Phys. Conf. Ser., vol. 92, no. 012068, pp. 1-4, 2007.

[37] J. J. Lim, S. Sujecki, and E. C. Larkins, "The influence of surface effects on the simulation of 1.3, $\mu \mathrm{m}$ InGaAsN edge-emitting lasers," presented at the NUSOD, Singapore, Sep. 11-14, 2006, Paper ThPD1.

[38] R. B. Darling, "Defect-state occupation, Fermi-level pinning, and illumination effects on free semiconductor surfaces," Phys. Rev. B, vol. 43, no. 5, pp. 4071-83, 1991.

[39] I. Vurgaftman, J. R. Meyer, and L. R. Ram-Mohan, "Band parameters for III-V compound semiconductors and their alloys," J. Appl. Phys., vol. 89, no. 11, pp. 5815-5875, 2001.

[40] J. J. Lim, R. MacKenzie, S. Sujecki, E. C. Larkins, M. Sadeghi, S. M. Wang, Y. Q. Wei, J. S. Gustavsson, A. Larsson, P. Melanen, P. Sipilä, P. Uusimaa, A. A. George, and P. M. Smowton, "Simulation of DQW GaInNAs laser diodes," IET Optoelectron., vol. 1, no. 6, pp. 259-265, 2007.

[41] K.-H. Hasler, B. Sumpf, P. Adamiec, F. Bugge, J. Fricke, P. Ressel, H. Wenzel, G. Erbert, and G. Tränkle, "5-W DBR tapered lasers emitting at $1060 \mathrm{~nm}$ with a narrow spectral linewidth and a nearly diffraction-limited beam quality," IEEE Photon. Technol. Lett., vol. 20, no. 19, pp. 16481650, Oct. 2008.

[42] B. W. Hakki and L. Paoli, "Gain spectra in GaAs double-heterostructure injection lasers," J. Appl. Phys., vol. 46, no. 3, pp. 1299-1306, 1975.

[43] D. T. Cassidy, "Technique for measurement of the gain spectra of semiconductor diode lasers," J. Appl. Phys., vol. 56, no. 11, pp. 3096-3099, 1984.

[44] P. Blood, G. M. Lewis, P. M. Smowton, H. Summers, J. Thomson, and J. Lutti, "Characterization of semiconductor laser gain media by the segmented contact method," IEEE J. Sel. Topics Quantum Electron., vol. 9, no. 5, pp. 1275-1282, Sep./Oct. 2003.

[45] M. Fukuda, Reliability and Degradation of Semiconductor Lasers and LEDs. Boston, MA: Artech House, 1991.

[46] N. C. Gerhardt, M. R. Hofmann, J. Hader, J. V. Moloney, S. W. Koch, and H. Riechert, "Linewidth enhancement factor and optical gain in (GaIn)(NAs)/GaAs lasers," Appl. Phys. Lett., vol. 84, no. 1, pp. 1-3, 2004.

[47] R. MacKenzie, J. J. Lim, S. Bull, S. Chao, S. Sujecki, M. Sadeghi, S. M. Wang, A. Larsson, P. Melanen, P. Sipila, P. Uusimaa, and E. C. Larkins, "Measurement of optical gain, effective group index and linewidth enhancement factor in $1.3 \mu \mathrm{m}$ dilute nitride doublequantum-well lasers," IET Optoelectron., vol. 1, no. 6, pp. 284-288, 2007.

[48] S. Bull, A. V. Andrianov, J. G. Wykes, J. J. Lim, S. Sujecki, S. C. Auzanneau, M. Calligaro, M. Lecomte, O. Parillaud, M. Krakowski, and E. C. Larkins, "Quantitative imaging of intracavity spontaneous emission distributions using tapered lasers fabricated with windowed n-contacts," Proc. Inst. Electr. Eng. Optoelectron., vol. 153, no. 1, pp. 2-7, Feb. 2006.

[49] A. E. Siegman and H. Y. Miller, "Unstable optical resonator loss calculations using the Prony method," Appl. Opt., vol. 9, no. 12, pp. 2729-2736, 1970.

[50] J. M. G. Tijero, H. Odriozola, L. Borruel, I. Esquivias, S. Sujecki, and E. C. Larkins, "Enhanced brightness of tapered laser diodes based on 
an asymmetric epitaxial design," IEEE Photon. Technol. Lett., vol. 19, no. 20, pp. 1640-1642, Oct. 2007.

[51] A. Al-Muhanna, L. J. Mawst, D. Botez, D. Z. Garbuzov, R. U. Martinelli, and J. C. Connolly, "High-power $(>10 \mathrm{~W})$ continuous-wave operation from $100 \mu \mathrm{m}$ arerture $0.97 \mu \mathrm{m}$ emitting Al-free diode lasers," Appl. Phys. Lett., vol. 73, no. 9, pp. 1182-1184, 1998.

[52] M. Krakowski, S. C. Auzanneau, F. Berlie, M. Calligaro, Y. Robert, O. Parillaud, and M. Lecomte, "1W high brightness index guided tapered laser at $980 \mathrm{~nm}$ using Al-free active region materials," Electron. Lett., vol. 39, no. 15 , pp. 1122-1123, 2003.

[53] B. Thestrup, M. Chi, and P. M. Petersen, "Lateral mode selection in a broad area laser diode by self-injection locking with a mirror stripe," Proc. SPIE, vol. 5336, pp. 38-44, 2004.

[54] G. Pauliat, N. Dubreuil, and G. Roosen, "Self-organizing laser cavities," in Photorefractive Materials and Their Applications 3: Applications (Springer Series in Optical Science), P. Günter and J.-P. Huignard, Eds. New York: Springer-Verlag, 2007, pp. 253-275.

[55] Z. Zhang, G. Pauliat, J. J. Lim, P. J. Bream, N. Dubreuil, A. J. Kent, E. C. Larkins, and S. Sujecki, "Numerical modeling of high-power selforganizing external cavity lasers," Opt. Quantum Electron., to be published.

[56] G. L. Bourdet, I. Hassiaoui, R. McBride, J. F. Monjardin, H. Baker, N. Michel, and M. Krakowski, "High-power, low-divergence, linear array of quasi-diffraction-limited beams supplied by tapered diodes," Appl. Opt., vol. 46, no. 25, pp. 6297-6301, 2007.

[57] D. Paboeuf, G. Lucas-Leclin, P. Georges, N. Michel, M. Krakowski, J. J. Lim, S. Sujecki, and E. C. Larkins, "Narrow-line coherently-combined tapered laser diodes in a Talbot external cavity with a volume Bragg grating," Appl. Phys. Lett., vol. 93, pp. 211102-1-211102-3, 2008.

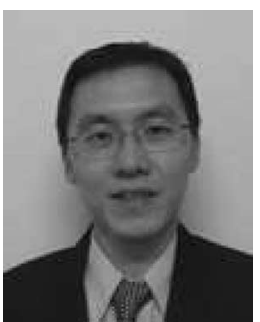

Jun Jun Lim (S'01-M'04) was born in Batu Pahat, Malaysia, in 1978. He received the B.Eng. degree (with first-class honors) in electronic engineering and the Ph.D. degree from the University of Nottingham, Nottingham, U.K., in 1999 and 2003, respectively.

Since 2004, he has been a Research Fellow at the University of Nottingham, where he was involved in several projects on the modeling of high-speed and high-brightness laser diodes. His current research interests include the simulation, analysis, and design of optoelectronic devices.

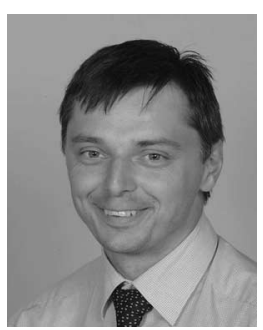

Slawomir Sujecki (M'05-SM'08) was born in Grojec, Poland, in 1969. He received the M.Sc. and Ph.D. degrees in electronic engineering from Warsaw University of Technology, Warsaw, Poland, in 1993 and 1997, respectively.

During 1994-1995, he was a Deutscher Akademischer Austauschdienst Fellow with the Technische Universität Berlin. During 1996-1998, he was a British Council Fellow and then a Royal Society/Wolfson Foundation Fellow at the University of Nottingham, Nottingham, U.K., where he joined the Department of Electrical and Electronic Engineering in April 2001. He started lecturing at Kielce University of Technology, Poland, in 1998. He joined the National Institute of Telecommunications, Poland, in 1999.

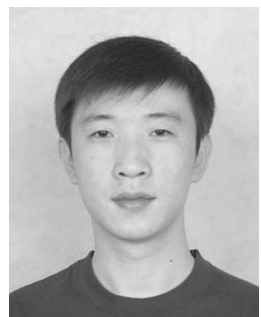

Lei Lang (S'07) was born in Shijiazhuang, China, in 1981. He received the B.Eng. degree in electronic information engineering from Hebei University of Technology, Tianjin, China, in 2004, and the M.Sc. degree in electronic communications and computer engineering in 2005 from the University of Nottingham, Nottingham, U.K., where he is currently working toward the Ph.D. degree in electrical and electronic engineering.

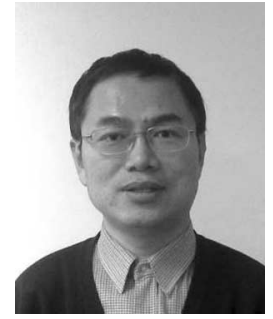

Zhichao Zhang received the B.Eng. and M.Sc. degrees in electrical and electronic engineering form Shandong University, Jinan, China, in 1987 and 1990 respectively. $\mathrm{He}$ is currently working toward the $\mathrm{Ph} . \mathrm{D}$. degree in electrical and electronic engineering at the University of Nottingham, Nottingham, U.K.

David Paboeuf was born in Nantes, France, in 1984. He received the Engineering degree from the Ecole Supérieure d'Optique, Orsay, France, and the Master's degree in optics and photonics from the University Paris Sud, Orsay, France, in 2006. He is currently working toward the Ph.D. at the Laboratoire Charles Fabry de l'Institut d'Optique, Palaiseau, France, on the development of external cavities for brightness improvement of laser diodes.

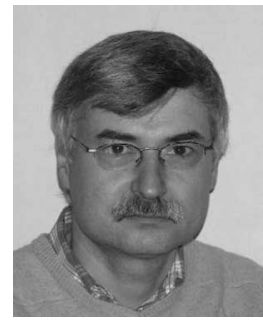

Gilles Pauliat received the Ph.D. degree from the University Paris Sud, Orsay, France, in 1986.

He was a Postdoctoral Researcher in the Quantum Electronic Laboratory, Federal Polytechnic School, Zurich, for one year. He joined the Centre National de la Recherche Scientifique, Palaiseau, France, where he is currently the Head of the Matériaux non Linéaires et Applications Research Group, Laboratoire Charles Fabry de l'Institut d'Optique. His current research interests include high-density data storage with holographic memories and self-organizing

laser cavities.

Dr. Pauliat is a member of the Board of Directors of the European Optical Society.

Gaëlle Lucas-Leclin was born in Guérande, France. She received the M.S. degree in optics from the Ecole Supérieure d'Optique, Orsay, France, in 1994, and the Ph.D. degree from the University Paris XI, Orsay, in 1998.

Since 1998, she has been an Assistant Professor at the Institut d'Optique Graduate School, Centre National de la Recherche Scientifique (CNRS), University Paris Sud, Palaiseau, France, where she is with the Laboratoire Charles Fabry de l'Institut d'Optique, and is in charge of the semiconductor physics and semiconductor sources lectures. Her current research interests include extended cavity laser diodes and vertical external cavity semiconductor lasers.

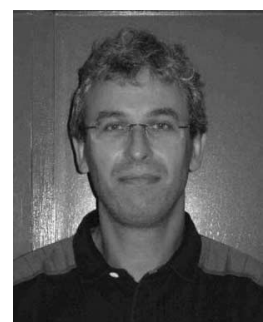

Patrick Georges was born in Metz, France, in 1962. He received the Engineering degree from the Ecole Supérieure d'Optique, Orsay, France, in 1985, and the Ph.D. degree on colliding pulses mode locked dye lasers at different wavelengths and pulse compression from the University of Paris XI, Orsay, in 1989.

$\mathrm{He}$ is currently a Senior Scientist at the Centre National de la Recherche Scientifique (CNRS), Palaiseau, France, where he is with the Institut d'Optique, and leads the Lasers and Biophotonics Group, Laboratoire Charles Fabry de l'Institut d'Optique. His current research interests include diode-pumped solid-state lasers, new laser materials, picosecond and femtosecond lasers, high-brightness laser diodes, fiber amplifier systems, and applications of picosecond or femtosecond lasers in biophotonics (time-resolved fluorescence spectroscopy, two-photon microscopy).

Dr. Georges is a Fellow of the Optical Society of America. 
Roderick C. I. MacKenzie received the M.Eng. and Ph.D. degrees in electronic engineering from the University of Nottingham, Nottingham, U.K., in 2004 and 2008 , respectively.

He was engaged in the measurement and simulation of $1.3-\mu \mathrm{m}$ dilute nitride laser diodes, focusing specifically on self-heating and its impact on device performance. He is currently with the University of Nottingham. His current research interests include measurement of device gain and thermographic imagery.

Philip Bream received the M.Eng. degree (with first-class honors) in electronic engineering and the Ph.D. degree from the University of Nottingham, Nottingham, U.K., in 2002 and 2006, respectively.

He was engaged in research on nonequilibrium carrier dynamics and gain in semiconductor quantum wells (QWs). He is currently with the University of Nottingham

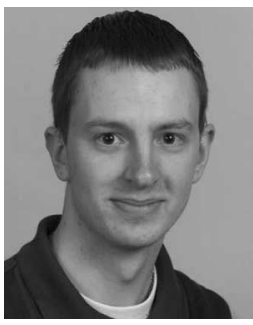

Stephen Bull (S'01-M'04) received the M.Eng. degree (with first-class honors) in electronic engineering with German and the Ph.D. degree from the University of Nottingham, Nottingham, U.K., in 2001 and 2004, respectively.

He is currently a Postdoctoral Research Fellow at the University of Nottingham. His current research interests include spectroscopic imaging techniques for the characterization of laser diodes, including external cavity lasers, the validation of simulation tools ics of high-power laser bars. using these techniques, and the degradation dynam-

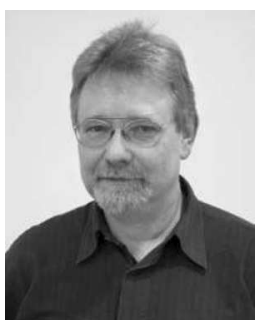

Karl-Heinz Hasler was born in Ludwigslust, Germany, in 1952. He received the Dipl.-Phys. degree from Humboldt University, Berlin, Germany, in 1978.

From 1978 to 1991, he was with the semiconductor industry, where he was involved in the development of LEDs, near-infrared response (NIR) detectors, and laser diodes. From 1992 to 2000, he was with the Astrophysikalisches Institut Potsdam (AIP), where he was involved in the field of magnetohydrodynamics and helioseismology. Since 2000 , he has been with the Ferdinand-Braun-Institut für Höchstfrequenztechnik, Berlin, where he is engaged in the development of high-power, high-brightness semiconductor lasers.

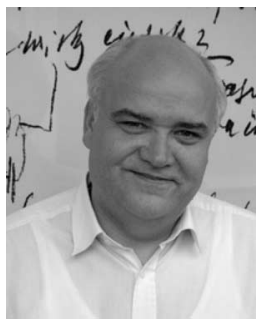

Bernd Sumpf was born in Berlin, Germany, in 1958 $\mathrm{He}$ received the Diploma in physics and the Ph.D degree from Humboldt-Universität Berlin, Berlin, in 1981 and 1987, respectively, and the Privat-Dozent degree in 1997.

$\mathrm{He}$ was engaged in research on lead salt diode lasers for spectroscopic applications. From 1993 till 1997, he was with the Technische Universität Berlin, where he was engaged in research on high-resolution spectroscopy and difference-frequency generation. Since 2000 , he has been with the Ferdinand-BraunInstitut für Höchstfrequenztechnik, Berlin, where he is involved in the field of high-power and high-brightness diode lasers.

Hans Wenzel received the Diploma and Doctoral degrees in physics from Humboldt University, Berlin, Germany, in 1986 and 1991, respectively.

$\mathrm{He}$ was engaged in research on electrooptical modeling of semiconductor lasers. From 1991 to 1994, he was involved in a research project on the 3D simulation of DFB lasers. In 1994, he joined the Ferdinand-Braun-Institut für Höchstfrequenztechnik, Berlin, where he is engaged in the development of high-brightness semiconductor lasers. His current research interests include the analysis, modeling, and simulation of optoelectronic devices.

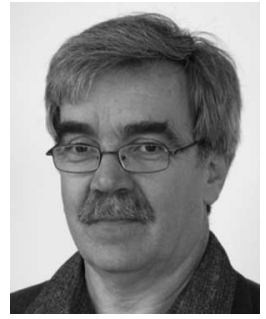

Götz Erbert (M'95) received the Diploma in physics from Humboldt University, Berlin, Germany, in 1973, and the Doctoral degree in physics from the Academy of Sciences, Berlin, German Democratic Republic (GDR), in 1990

From 1973 to 1991, he was with the Academy of Sciences, where he was first engaged in the field of integrated optics and dynamic holographic gratings in semiconductors, and later in semiconductor lasers. In 1992, he joined the Ferdinand-Braun-Institut für Höchstfrequenztechnik, Berlin, where he has been engaged in the optoelectronic activities since 1996. He is also involved in research on high-power semiconductor lasers based on GaAs using strainedlayer quantum-well active regions in the wavelength range from 650 to 1200 $\mathrm{nm}$

Birgitte Thestrup received the Ph.D. degree in physics from the University of Copenhagen, Copenhagen, Denmark, in 1999.

She is currently a Senior Scientist in the Department of Photonics Engineering, Technical University of Denmark, Roskilde, Denmark. From 2001 to 2007 , she was with the Diode Laser Group, Optics and Plasma Research Department, Risoe National Laboratory, Denmark, where she also worked on laser-induced plasmas. She is the author or coauthor of more than 30 papers and one book. She holds two patents. Her current research interests include external cavity diode laser systems for industrial and medical applications, and high-quality and energy-efficient solid-state lighting systems for specific applications.

Paul Michael Petersen received the M.Sc. degree in engineering and the Ph.D. degree in physics from the Technical University of Denmark (DTU), Roskilde, Denmark, in 1983 and 1986, respectively.

He has 15 years of research experience in laser physics, nonlinear optics, and optical measurement techniques, and has headed several collaborative research projects within laser physics. He is currently the Head of light sources and industrial sensors in the Department of Photonics Engineering, DTU. He is also an Adjunct Professor of optics at Niels Bohr Institute, Copenhagen University, Copenhagen, Denmark. He is the Head of the Center for Biomedical Optics and New Laser Systems at DTU. He has authored or coauthored more than 100 scientific publications and nine patents. He has 20 years of teaching experience in optics, quantum optics, laser technique, and nonlinear optics.

Nicolas Michel, photograph and biography not available at the time of publication.

Michel Krakowski, photograph and biography not available at the time of publication.

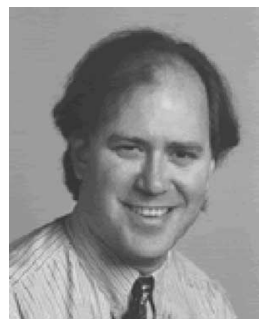

Eric C. Larkins (S'82-M'89-SM'07) received the B.S.E.E. degree from Cornell University, Ithaca, NY, in 1980, and the M.S.E.E. degree and the Ph.D. degree in electrical engineering from Stanford University, Stanford, CA, in 1985 and 1991, respectively.

In 1994, joined the Department of Electrical and Electronic Engineering, University of Nottingham, Nottingham, U.K., where he became a Professor of optoelectronics in 2002. From 1991 to 1994, he was a Visiting Scientist at the Fraunhofer Institut für Angewandte Festkörperphysik, Freiburg, Germany. His current research interests include laser diodes, and functional and nanophotonic devices. 